\title{
UNIVERSITYOF BIRMINGHAM

\section{Activation of the FGFR1 signalling pathway by the Epstein-Barr virus-encoded LMP1 promotes aerobic glycolysis and transformation of human nasopharyngeal epithelial cells}

Lo, Angela Kwok-Fung; Dawson, Christopher W; Young, Lawrence S; Ko, Chuen-Wai; Hau, Pok-Man; Lo, Kwok-Wai

DOI:

10.1002/path.4575

License:

None: All rights reserved

Document Version

Peer reviewed version

Citation for published version (Harvard):

Lo, AK-F, Dawson, CW, Young, LS, Ko, C-W, Hau, P-M \& Lo, K-W 2015, 'Activation of the FGFR1 signalling pathway by the Epstein-Barr virus-encoded LMP1 promotes aerobic glycolysis and transformation of human nasopharyngeal epithelial cells', Journal of Pathology, vol. 237, no. 2, pp. 238-248.

https://doi.org/10.1002/path.4575

Link to publication on Research at Birmingham portal

Publisher Rights Statement:

Checked for eligibility: 01/04/2015. This is the peer reviewed version of the following article :Lo, A. K.-F., Dawson, C. W., Young, L. S., Ko, C.-W., Hau, P.-M. and Lo, K.-W. (2015), Activation of the FGFR1 signalling pathway by the Epstein-Barr virus-encoded LMP1 promotes aerobic glycolysis and transformation of human nasopharyngeal epithelial cells. J. Pathol., 237: 238-248. doi: 10.1002/path.4575. , which has been published in final form at DOI: $10.1002 /$ path.4575. This article may be used for non-commercial purposes in accordance with Wiley Terms and Conditions for Self-Archiving.

\footnotetext{
General rights

Unless a licence is specified above, all rights (including copyright and moral rights) in this document are retained by the authors and/or the copyright holders. The express permission of the copyright holder must be obtained for any use of this material other than for purposes permitted by law.
}

- Users may freely distribute the URL that is used to identify this publication.

- Users may download and/or print one copy of the publication from the University of Birmingham research portal for the purpose of private study or non-commercial research.

- User may use extracts from the document in line with the concept of 'fair dealing' under the Copyright, Designs and Patents Act 1988 (?)

- Users may not further distribute the material nor use it for the purposes of commercial gain.

Where a licence is displayed above, please note the terms and conditions of the licence govern your use of this document.

When citing, please reference the published version.

Take down policy

While the University of Birmingham exercises care and attention in making items available there are rare occasions when an item has been uploaded in error or has been deemed to be commercially or otherwise sensitive.

If you believe that this is the case for this document, please contact UBIRA@lists.bham.ac.uk providing details and we will remove access to the work immediately and investigate. 
Title: Activation of the FGFR1 signalling pathway by the Epstein-Barr Virus-encoded LMP1 promotes Aerobic Glycolysis and Transformation of Human Nasopharyngeal Epithelial cells

Angela Kwok-Fung Lo ${ }^{1,2}$, Christopher W. Dawson'², Lawrence S. Young ${ }^{3}$, Chuen-Wai Ko', Pok-Man Hau ${ }^{1}$, and Kwok-Wai Lo ${ }^{{ }^{*}}$

${ }^{1}$ Department of Anatomical and Cellular Pathology, State Key Laboratory in Oncology in South China and Li Ka Shing Institute of Health Science, Prince of Wales Hospital, The Chinese University of Hong Kong, Hong Kong

${ }^{2}$ Cancer Research UK Cancer Centre, School of Cancer Sciences, University of Birmingham, Vincent Drive, Edgbaston, Birmingham, B15 2TT, UK

${ }^{3}$ Warwick Medical School, University of Warwick, Coventry, CV4 7AL, UK

*Corresponding author: Kwok-Wai Lo

Department of Anatomical and Cellular Pathology, Prince of Wales Hospital,

The Chinese University of Hong Kong, Shatin, N.T., Hong Kong

Tel: $852-2632$ 2178, Fax: $852-26376274$

Email: kwlo@cuhk.edu.hk

Short Running Title: Activation of FGFR1 Signal Pathway by EBV LMP1

Conflict of Interest statement: The authors declare that there are no conflicts of interest.

Total Word No.: 3999 (Introduction to Discussion) 


\begin{abstract}
Non-keratinizing nasopharyngeal carcinoma (NPC) is closely associated with Epstein-Barr virus (EBV) infection. The EBV-encoded latent membrane protein 1 (LMP1) is believed to play an important role in NPC pathogenesis by virtue of its ability to activate multiple cell signalling pathways which collectively promote cell proliferation, transformation, angiogenesis and invasiveness as well as modulation of energy metabolism. In this study, we report that LMP1 increases cellular uptake of glucose and glutamine, enhances LDHA activity and lactate production, but reduces pyruvate kinase activity and pyruvate concentrations. LMP1 also increases the phosphorylation of PKM2, LDHA and FGFR1 as well as the expression of PDHK1, FGFR1, c-Myc and HIF-1 $\alpha$ regardless of oxygen availability. Collectively, these findings suggest that LMP1 promotes aerobic glycolysis. With respect to FGFR1 signalling, LMP1 not only increases FGFR1 expression, but also upregulates FGF2, leading to constitutive activation of the FGFR1 signalling pathway. Furthermore, two inhibitors of FGFR1 (PD161570 and SU5402) attenuate LMP1-mediated aerobic glycolysis, cellular transformation (proliferation and anchorage-independent growth), cell migration and invasion in nasopharyngeal epithelial cells, identifying FGFR1 signalling as a key pathway in LMP1-mediated growth transformation. Immunohistochemical staining revealed that high levels of phosphorylated FGFR1 are common in primary NPC specimens, and that this correlated with the expression of LMP1. In addition, FGFR1 inhibitors suppress cell proliferation and anchorage-independent growth of NPC cells. Our current findings demonstrate that LMP1-mediated FGFR1 activation contributes to aerobic glycolysis and transformation of epithelial cells, thereby implicating FGF2/FGFR1 signalling activation in the EBV-driven pathogenesis of NPC.
\end{abstract}

Key words: Nasopharyngeal Carcinoma; Epstein-Barr virus; Glycolysis; LMP1; FGFR1 


\section{Introduction}

Non-keratinizing undifferentiated nasopharyngeal carcinoma (NPC) is closely associated with EpsteinBarr virus infection. The EBV-encoded LMP1 protein, which is commonly expressed in NPC, is of particular interest, as it displays oncogenic properties in vivo and in vitro [1]. LMP1 engages multiple signalling cascades that include the NF-кB, PI3K-AKT, ERK-MAPK, JNK, JAK-STAT, and p38/MAPK pathways to alter various gene expression programs [1;2]. LMP1 is essential for EBV immortalisation of primary B cells [3]. In epithelial cells, LMP1 promotes cell proliferation, anchorage independent growth, cell survival, angiogenesis, cell invasion and migration. LMP1 also dysregulates energy metabolism. These observations suggest that LMP1 is a prime candidate for driving NPC development [1-3].

Cellular transformation is characterised by reduced oxidative phosphorylation and increased aerobic glycolysis, where cells rapidly increase glucose utilization and lactate production regardless of oxygen availability [4]. Aerobic glycolysis facilitates rapid cell division by providing both energy and metabolic intermediates for the anabolic biosynthesis of macromolecules [4]. Accumulating evidence indicates that HIF-1 and c-Myc play important roles in aerobic glycolysis by altering the activities of multiple metabolic enzymes that include glucose transporter 1 (GLUT1), hexokinase 2 (HK2), lactate dehydrogenase (LDHA), pyruvate dehydrogenase kinase 1 (PDHK1) and pyruvate kinase M2 isoform (PKM2) [4-6]. In addition to HIF-1 $\alpha$ and c-Myc, fibroblast growth factor 1 (FGFR1) is also found to play an important role in aerobic glycolysis $[7 ; 8]$.

The FGFR family consists of four receptor tyrosine kinases (FGFR1-4). Upon FGF-ligand binding, FGFRs undergo tyrosine autophosphorylation, activating various signalling cascades, which include the MEK/ERK-MAPK and PI3K/Akt pathways. FGFR activation facilitates cell proliferation, survival, invasion, migration and angiogenesis. In vitro studies have demonstrated that over-activation of FGFR1 in non-transformed mammary cells results in morphological transformation. Also, the induction of FGFR1 in normal human urothelial cells promotes cell proliferation and cell survival [9]. Furthermore, FGFR1 promotes aerobic glycolysis by inactivating PKM2, while enhancing the activities of LDHA1 and PDHK1 [7;8;10;11]. In this study, we report that LMP1 activates FGF2/FGFR1 signalling, an effect that contributes to LMP1-mediated aerobic glycolysis, cell proliferation, anchorage-independent growth, migration and invasion. Immunohistochemical analysis revealed that FGFR1 activation is common in primary NPC and correlated with the expression of LMP1. Our findings demonstrate that LMP1-mediated FGFR1 activation contributes to tumour progression, implicating the involvement of FGF2/FGFR1 signalling in the pathogenesis of NPC. 


\section{Materials and methods}

\section{Cell lines, chemical, drug and transfection}

C666-1 and SCC12F cells were maintained in RPMI 1640 and DMEM/F12 medium respectively. NP69 cells were maintained in keratinocyte-serum free medium (Life Technologies, Grand Island, NY, USA). PD161570 and SU5402 were from Sigma, St Louis, MO, USA. Cell transfection was performed using Fugene HD (Promega, Madison, WI, USA). LMP1 siRNA was from Dharmacon Inc., Lafayette, CO, USA. Details are described in the Supplementary materials and methods.

\section{Biochemical assays}

Colorimetric assays were performed according to manufacturer's protocol. The Lactate Assay Kit, Glutamine and Glutamate Kit, and Lactate Dehydrogenase Activity Kit were from Sigma. The Glucose Assay Kit, Pyruvate Assay Kit and Pyruvate Kinase Assay Kit were from Abcam. The Amplex® Red Glutamic Acid/Glutamate Oxidase Assay Kit were from Life Technologies. Details are described in the Supplementary materials and methods.

\section{Quantitative RT-PCR}

All QRT-PCR products were amplified using Power SYBR green PCR Master Mix Kit (Life Technologies). Details are outlined in the Supplementary materials and methods.

\section{Luciferase reporter assay}

Luciferase reporter assays were performed using the Dual-Luciferase Reporter Assay System (Promega). Details are outlined in the Supplementary materials and methods.

\section{Western blotting analysis}

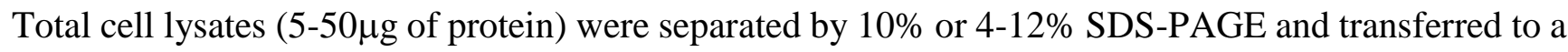
PVDF membrane prior to immunoblotting. Antibodies to LMP1 were purchased from Dako, Glostrup, Denmark and $\alpha$-tubulin from Santa Cruz, Dallas, TX, USA. All other antibodies were from Cell Signalling Technology (Beveley, MA, USA).

\section{Immunofluorescence staining}

Immunofluorescence staining was performed as previously described [12]. Details are outlined in the Supplementary.

\section{Immunohistochemical staining}

Immunohistochemical staining was performed as described previously [13]. Details are outlined in the Supplementary materials and methods. 


\section{Cell Proliferation assay}

Cell proliferation assay was performed with cell proliferation reagent CCK-8 (Dojindo Molecular Technologies, Rockvilles, MD, USA). Details are outlined in the Supplementary.

\section{Soft Agar Cloning Assay}

Soft agar colony formation assays were performed as previously described [14;15]. Details are outlined in the Supplementary.

\section{Migration and Invasion Assays}

Cell migration assays were performed using CytoSelect 24-well Wound Healing Assay Kit (Cell Biolabs, San Diego, CA, USA). Boyden chamber invasion assays were performed using the CytoSelect 24-Well Cell Invasion Assay Kit (Cell Biolabs), respectively. For collagen gel invasion assays, a collagen mixture was prepared with type I collagen solution (Life Technologies). Details are outlined in the Supplementary.

\section{Statistical Analysis}

Where applicable, the significance of difference between two groups was evaluated with an unpaired two-tailed student's t-test. The IC 50 values of PD161570 and SU5402 were determined using GraphPad Prism software by applying the four-parameter logistic equation to generate the Sigmoidal doseresponse (variable slope) curves.

\section{Results}

LMP1 promotes aerobic glycolysis

To examine the effects of LMP1 on the glycolytic pathway, we firstly examined lactate production in control (NP69-pLNSX) and LMP1 expressing NP69 epithelial cells (NP69-LMP1). As shown in Figure 1A, increased levels of lactate production (approximately 1.4-fold higher) were observed in NP69LMP1 cells compared to NP69-pLNSX cells at day 7. We also found that LDHA activity in NP69LMP1 cells was 2.3-fold higher than that in NP69-pLNSX cells (Figure 1B). Pyruvate kinase (PK) catalyses the conversion of PEP to pyruvate and ATP. Inactive PK promotes aerobic glycolysis $[4 ; 8 ; 16]$. Here, we demonstrate that PK activity was reduced by 20\% in NP69-LMP1 cells (Figure 1C) and the intracellular concentration of pyruvate in NP69-LMP1 cells was 22\% lower than that in NP69-pLNSX cells (Figure 1D). Aerobic glycolysis is associated with a high rate of glucose uptake. In agreement with a recent study on NPC cells [17], the uptake of glucose in NP69-LMP1 cells was increased by approximately 1.33 -fold (Figure 1F). Glucose uptake is facilitated by glucose transporters (GLUT). Using immunofluorescence staining, Sommermann $\mathrm{T}$ et al showed that LMP1 promotes the 
translocation of GLUT1 from intracellular vesicles to the plasma membrane in B lymphocytes [18]. Here, compared to NP69-pLNSX cells, where GLUT1 was mainly found in intracellular membrane compartments, GLUT1 was found in the plasma membrane of NP69-LMP1 cells (Figure 1E \& Supplementary Figure 1). This finding confirms that LMP1 promotes GLUT1 translocation to the plasma membrane in nasopharyngeal epithelial cells, facilitating higher rates of glucose transport. In addition to glucose, glutamine is another carbon source essential for cell growth. Here, we found that NP69-LMP1 cells had a 1.67-fold higher level of glutamine consumption compared to NP69-pLNSX cells (Figure 1G). The intracellular concentration of glutamate was also higher in NP69-LMP1 compared to NP69-pLNSX (approximately 1.24-fold) (Figure 1H). Overall, LMP1 increased the uptake of glucose and glutamine, enhanced lactate production and reduced pyruvate kinase activity, suggesting that LMP1 promotes aerobic glycolysis (Supplementary Figure 6).

Next, we examined the expression of glycolytic pathway-associated proteins. As shown in Figure 2A, LMP1 induced the levels of Tyr10 phosphorylated-LDHA and Tyr105 phosphorylated-PKM2 as well as the total amounts of LDHA and PDHK1 protein, in a dose dependent manner. However, LMP1 had no effect on the expression of PKM2 and pyruvate dehydrogenase A1 (PDHA1). PKM2 phosphorylation at Tyr105 is associated with decreased enzymatic activity, while LDHA phosphorylation at Tyr10 upregulates LDHA activity, promoting aerobic glycolysis [7;10]. PDHA1 is negatively regulated by PDHK1. Inactivation of PDHA1 by PDHK1 leads to decreased conversion of pyruvate to acetyl-CoA, allowing more pyruvate to convert to lactate [8]. Accordingly, LMP1 promotes aerobic glycolysis by inactivating PKM2 and PDHA1 while enhancing the activities of LDHA and PDHK1 (Supplementary Figure 6). Also, LMP1 alters the expression and activity of glycolytic proteins under both normoxic and hypoxic conditions (Figure 2B), suggests that LMP1 induces aerobic glycolysis regardless of oxygen availability. Aerobic glycolysis is regulated by numerous oncoproteins that include HIF-1 and c-Myc. Previous studies have shown that HIF-1 and c-Myc are downstream targets of LMP1 [17;19-23]. Here, we find the increased expression of both HIF-1 $\alpha$ and c-Myc in NP69-LMP1 cells (Figure 2A \& 2B). Interestingly, LDHA, PKM2 and PDHK1 are transcriptional targets of c-Myc and HIF-1 [4-6]. Upregulation of LDHA and PDHK1 by LMP1 may be mediated through LMP1-induced c-Myc and HIF-1 activity. However, this is still not clear as to the mechanism by which LMP1 induces the phosphorylation of LDHA and PKM2.

\section{LMP1 induces expression and activation of FGFRI}

Previous studies have shown that FGFR1 directly phosphorylates LDHA at Tyr10 and PKM2 at Tyr105 to enhance LDHA activity and inactivate PKM2 respectively [7]. Thus, we sought to examine the effect of LMP1 on FGFR1 expression. As shown in Figure 2A \& B, we found that LMP1 increased the levels 
of total and phosphorylated FGFR1 protein. Similar results were observed in LMP1 expressing squamous carcinoma SCC12F (SCC12F-LMP1) cells (Supplementary Figure 2). By quantitative RTPCR, we found that LMP1 augmented FGFR1 mRNA expression in a dose dependent manner (Figure 2C \& D). In both NP69-LMP1 and SCC12F-LMP1 cells, high levels of FGFR1 mRNA were observed. The expression of LMP1 and FGFR1 mRNA was also observed in EBV-infected C666-1 NPC cells (Supplementary Figure 3A \& 3B). Using a luciferase reporter assay, we found that LMP1 stimulated FGFR1 promoter activity in a dose dependent manner (Figure 2E). LMP1 has been reported to induce FGF2, a ligand for FGFR1 [24]. We also found that LMP1 stimulated FGF2 expression in nasopharyngeal epithelial cells (Figure 2F). High levels of FGF2 mRNA were also observed in NP69LMP1 and C666-1 cells (Supplementary Figure 3C). Our findings suggest a role for LMP1 in constitutively activating FGF2/FGFR1 signalling pathway by upregulating both FGFR1 and FGF2. Using a FGF2 specific neutralizing antibody, we found that inhibiting the binding of FGF2 to FGFR1 led to a robust reduction in FGFR1 phosphorylation in NP69-LMP1 cells (58\%) compared to NP69pLNSX cells (20\%) (Figure 2G). This finding indicates that activation of FGFR1 by LMP1 is mediated, predominantly through LMP1-upregulation of FGF2. In C666-1 cells, which express low levels of LMP1 protein [12;25], siRNA knock down of LMP1 resulted in a reduction in the levels of total and phosphorylated FGFR1 proteins (Figure 2H), further confirming the activation of FGFR1 by LMP1 in EBV-infected NPC cells.

Activation of FGFR1 signalling by LMP1 facilitates aerobic glycolysis

To determine whether LMP1-mediated FGFR1 activation contributed to aerobic glycolysis, NP69pLNSX and NP69-LMP1 cells were treated with PD16157 (PD) or SU5402 (SU), two specific FGFR1 inhibitors which suppress FGFR1 tyrosine kinase phosphorylation. As shown in Figure 3A, FGFR1 inhibitors suppressed FGFR1 phosphorylation in all cell lines examined; however, the suppression was more obvious in NP69-LMP1 cells compared to NP69-pLNSX cells. Although treatment with 1 $\mu$ M PD resulted in no significant change in FGFR1 phosphorylation in NP69-pLNSX cells, a 50\% reduction was observed in NP69-LMP1 cells. FGFR1 inhibitors also suppressed LMP1-induced LDHA phosphorylation and PDHK1 protein expression. Treatment with $1 \mu \mathrm{M}$ PD reduced the level of LDHA phosphorylation and PDHK1 protein by 50\% and 40\% respectively in NP69-LMP1 cells, while there was no significant change in NP69-pLNSX cells. However, a high dose of PD $(5 \mu \mathrm{M})$ inhibited phosphorylation or the expression of FGFR1, LDHA, and PDHK1 in both NP69-pLNSX and NP69LMP1 cells. Similar results were observed when cells were treated with SU5402 (Figure 3A). Furthermore, both FGFR1 inhibitors reduced HIF-1 $\alpha$ expression, particularly in NP69-LMP1 cells. In response to $1 \mu \mathrm{M}$ PD treatment, the expression of HIF-1 $\alpha$ in NP69-LMP1 and NP69-pLNSX cells was reduced by $85 \%$ and $20 \%$ respectively. This finding suggests that LMP1-mediated FGFR1 signalling, 
partially if not completely, upregulates HIF-1 $\alpha$ expression. The ERK-MAPK and AKT pathways are activated in response to FGFR stimulation [26;27]. In response to PD and SU, a reduction in Akt and Erk1/2 phosphorylation was observed in both NP69-pLNSX and NP69-LMP1 cells (Figure 3A). Although PKM2 has been reported to be a downstream target of FGFR1 [7], we did not observe any significant inhibitory effect of PD161570 and SU5402 on PKM2 phosphorylation or protein expression in all cell lines examined. (Figure 3A).

Next, we examined the effects of FGFR1 inhibitors on the activities of LDHA and PK. As shown in Figure 3B, in the absence of FGFR1 inhibitors, LDHA activity in NP69-LMP1 cells was 2.2-fold higher than that of NP69-pLNSX cells. In the presence of 5 $\mu \mathrm{M}$ PD or $20 \mu \mathrm{M}$ SU, the LDHA activity in NP69LMP1 cells was reduced by $28 \%$ and $36 \%$ respectively, indicating that suppression of FGFR1 signalling attenuates LMP1-induced LDHA activity. Both FGFR1 inhibitors had little or no effect on PK activity in either NP69-pLNSX or NP69-LMP1 cells (Figure 3C), findings that are in agreement with results obtained by western blot analysis of PKM2 protein and phosphorylation (Figure 3A). Overall, these findings suggest that activation of FGFR1 by LMP1 alters the activity of glycolytic proteins, especially LDHA, thereby facilitating aerobic glycolysis. Interestingly, our current data did not support a previous report identifying PKM2 as a downstream target of FGFR1 [7].

\section{FGFRl activation is common in primary NPC tumours}

Next, we sought to examine the activity of FGFR1 in NPC tumours. Immunohistochemical (IHC) staining for phospho-FGFR1 (Tyr654) was performed on 42 NPC primary tumours. This set of samples has been examined in a previous study, where 12/42 were found to express LMP1 protein [13]. The intensity of phospho-FGFR1 staining was scored and a graph of the statistical dot plot of phosphoFGFR1 staining intensity against LMP1 expression was generated (Figure 4F). IHC staining revealed negative or weak expression of phospho-FGFR1 (immunoactivity score <3) in normal nasopharyngeal epithelium (NP) (Figure 4A) and in 19/42 (45\%) of NPC tumours (Figure 4B, representative NPC). In contrast, moderate or high levels of phosphorylated FGFR1 (immunoactivity score $\geq 3$ ) were detected in 23/42 NPC tumours (55\%) (Figure 4C-4E, representative NPCs). In particular, LMP1-positive NPC tumours were significantly associated with the higher levels of phosphorylated FGFR1 $(\mathrm{p}<0.005)$. (Figure 4F). These findings indicated that FGFR1 activation is common in NPC and correlates with LMP1 expression. Given that 40\% (12/30) of LMP1-negative tumours exhibited high levels of FGFR1 phosphorylation (Figure 4F). Other mechanisms, in addition to LMP1, may be involved in FGFR1 activation in NPC.

LMP1-induced FGFR1 signalling promotes cell growth and transformation 
To determine whether increased FGFR1 activity conferred a growth advantage to LMP1-exprssing cells, NP69-pLNSX and NP69-LMP1 cells were treated with increasing doses of PD or SU, and the effects on cell growth examined. As shown in Figure 5A \& 5B, suppression of FGFR1 activity reduced the proliferation of both NP69-pLNSX and NP69-LMP1 cells; however, NP69-LMP1 cells were more sensitive to FGFR1 inhibitors. At $2.5 \mu \mathrm{M}$ PD, the growth of NP69-pLNSX and NP69-LMP1 cells was reduced by $15 \%$ and $35 \%$ respectively ( $<<0.001$ ), while $20 \mu \mathrm{M}$ SU reduced cell proliferation of NP69pLNSX and NP69-LMP1 by $38 \%$ and 64\% respectively (p<0.001). Similar growth inhibitory responses were observed in SCC12F-LMP1 cells (Supplementary Figure 4). PD and SU also suppressed the growth of C666-1 NPC cells; the $\mathrm{IC}_{50}$ of PD at day 3 and SU at day 5 were $2.9 \mu \mathrm{M}$ and $9 \mu \mathrm{M}$ respectively (Figure 5C \& 5D). These findings indicate that suppression of FGFR1 activity inhibits LMP1-induced proliferation in non-malignant epithelial cells as well as the proliferation of LMP1 positive NPC cells, and implicates FGFR1 activation by LMP1 as an important mechanism in facilitating cell proliferation. Next, NP69-pLNSX and NP69-LMP1 cells were analysed for anchorage-independent growth. In agreement with our previous data [14;15], NP69-pLNSX cells failed to grow in soft agar irrespective of drug treatment (data not shown). In contrast, while NP69-LMP1 cells formed soft agar colonies under drug-free conditions, they were susceptible to FGFR1 inhibitors. $2 \mu \mathrm{M}$ PD reduced NP69-LMP1 colony formation by $71 \%$ whereas $15 \mu \mathrm{M}$ PD completely suppressed colony formation. $5 \mu \mathrm{M}$ and $20 \mu \mathrm{M}$ SU reduced colony formation of NP69-LMP1 cells by $19 \%$ and 71\% respectively (Figure 5E). We next examined the anchorage-independent growth of C666-1 NPC cells. As shown in Figure 5E, PD was more effective than SU in suppressing the colony formation of C666-1 cells, with $2 \mu \mathrm{M}$ and $15 \mu \mathrm{M}$ PD reducing soft agar colony by $71 \%$ and $99 \%$ respectively, while $5 \mu \mathrm{M}$ and $20 \mu \mathrm{M}$ SU resulted in $9 \%$ and $74 \%$ reduction. Overall, these findings suggest that LMP1-induced FGFR1 activity contributes to LMP1-mediated cellular transformation.

\section{LMP1-induced FGFR1 signalling promotes cell migration and invasion}

Our previous studies have shown that LMP1 promotes cell invasion and migration [14;15]. Here, we sought to determine whether inhibition of FGFR1 activity attenuated these responses. In wound healing assays (Figure 6A), NP69-pLNSX cells failed to migrate under drug-free conditions. Under the same conditions, NP69-LMP1 cells migrated into the wound area away from the edge, indicating increased motile response of NP69-LMP1 cells. However, in the presence $5 \mu \mathrm{M}$ PD or $10 \mu \mathrm{M}$ SU, the ability of NP69-LMP1 to migrate was virtually abolished. In collagen gel invasion assays (Figure 6B), NP69pLNSX cells formed spherical and non-invasive structures within the collagen gel matrix in the presence or absence of FGFR1 inhibitors, while, NP69-LMP1 cells formed long invasive branching tubules. However, in the presence of $2 \mu \mathrm{M}$ PD or $5 \mu \mathrm{M}$ SU, NP69-LMP1 cells exhibited blunt-ended and short tubules, indicating a reduction in cell invasion. In Boyden chamber invasion assays (Figure 6C), 
invading cells located at the bottom of the membrane were stained and imaged (Supplementary Figure 5). Stained cells were then quantified after stain extraction. Under drug-free condition, NP69-LMP1 displayed 2- to 2.4-fold higher invasive ability than that of NP69-pLNSX. However, in the presence of FGFR1 inhibitors, the invasive ability of NP69-LMP1was dramatically reduced. With 10 $\mu$ M PD or $20 \mu \mathrm{M}$ SU treatment, the invasive ability of NP69-LMP1 was reduced by $65 \%$ and $59 \%$ respectively. In contrast, $10 \mu \mathrm{M}$ PD or $20 \mu \mathrm{M}$ SU treatment only suppressed the invasive ability of NP69-pLNSX cells by $40 \%$ and $36 \%$, respectively. Overall, our data suggest that LMP1-mediated FGFR1 activation facilitates cell migration and invasion.

\section{Discussion}

LMP1 is crucial in EBV transformation of primary B cells, generating immortalized lymphoblastoid cell lines (LCLs) in which aerobic glycolysis is active [28]. In non-malignant nasopharyngeal epithelial cells, LMP1 induces cell proliferation, anchorage-independent growth, cell migration and invasion [14;15]. LMP1 also deregulates cellular metabolism by inactivating LKB1-AMPK signalling [13]. Whilst, the impact of LMP1 on glycolytic pathways has not been fully explored. LMP1 has been shown to increase the expression and activity of c-Myc and HIF-1 $\alpha$, two key regulators of glycolytic metabolism [17;19-23]. Here, we show that activation of the FGF2/FGFR1 signalling pathway by LMP1 not only promotes aerobic glycolysis, but also cell proliferation and transformation as well as migration and invasion (Figure 7).

In EBV immortalized LCLs, the concentration of lactate and pyruvate as well as LDHA activity were found to be significantly higher compared to mitogen-activated B cells, suggesting that aerobic glycolysis is active in LCLs [28]. Here, in nasopharyngeal epithelial cells, we show that LMP1 promotes aerobic glycolysis by increasing the uptake of glucose and glutamine, enhancing LDHA activity and lactate production, and by reducing PK activity and intracellular pyruvate concentrations (Figure 1). Interestingly, NP69-LMP1 cells have lower pyruvate concentrations compared to NP69-pLNSX cells, findings that differ to LCLs, which produce high levels of pyruvate [28]. The intracellular concentration of pyruvate is regulated predominantly by three glycolytic enzymes which include PK which converts PEP to pyruvate, LDHA which converts pyruvate to lactate in the cytosol and PDHA1 which converts pyruvate to acetyl-CoA in the mitochondria [8;29]. Thus, the overall concentration of pyruvate relies on the level and activities of these enzymes (Supplementary Figure 6). In NP69 and SCC12F cells, we found that LMP1 increases the phosphorylation of LDHA at Tyr10 and PKM2 at Tyr105 and the levels of LDHA and PDHK1 protein (Figure $2 \&$ Supplementary Figure 2). LDHA phosphorylation at Try 10 is necessary for LDHA activity, while, PKM2 phosphorylation at Tyr105 results in PKM2 inactivation and PDHK1 upregulation [8,11]. The inactivation of PKM2 by LMP1 reduces pyruvate production but 
increases the accumulation of upstream metabolic intermediates for biomass synthesis. Moreover, PDHK1 functions to phosphorylate and inactivate PDHA1 activity. Activation of LDHA and PDHK1 by LMP1 inhibits oxidation of pyruvate to Acetyl-CoA but promotes conversion of pyruvate to lactate. These observations suggest that LMP1 switches cellular oxidative phosphorylation to aerobic glycolysis (Supplementary Figure 6). The increased uptake of glutamine by LMP1 may contribute to glutaminolysis which is regulated by c-Myc [30]. Since c-Myc expression can be induced by LMP1 $[17 ; 19 ; 21 ; 23]$, the impact of LMP1 on glutaminolysis is worthy of further investigation.

In this study, we show that LMP1 increases FGFR1 expression in nasopharyngeal epithelial cells (Figure 2). Promoter luciferase reporter assays and qRT-PCR analysis indicate that LMP1 induces FGFR1 expressing by stimulating FGFR1 transcription (Figure 2C-E). The FGFR1 promoter is positively regulated by Sp1 and E2F-1 [31;32]. The activity of Sp1 is regulated by various kinases that include cyclin-dependent kinase (CDK), atypical protein kinase $\mathrm{C}-\zeta(\mathrm{PKC}-\zeta)$ and ERK-MAPK [33]. LMP1 has been reported to upregulate and/or activate CDK2, PKC- $\zeta$, and ERK kinases [1;34;35]. On the other hand, the transcriptional activity of E2F-1, which is inhibited by RB interaction, is activated in response to RB phosphorylation by cyclin D1. The induction of RB phosphorylation and cyclin D1 upregulation by LMP1 is mediated through NFאB, EGFR and STAT3 signalling [36-38]. As such, LMP1 induction of FGFR1 expression appeared to be mediated through multiple downstream targets and/or signal pathways (Supplementary Figure 7). Induction of FGF-2 by LMP1-mediated NFKB activation has been indicated [24]. We also found the upregulation of FGF2 in LMP1 expressing cells (Figure 2F). Blocking the binding of FGF-2 to FGFR1 with a FGF-2 neutralising antibody resulted in reduction of FGFR1 phosphorylation in NP69-LMP1 cells (Figure 2G), supporting a role for FGF2 ligand in driving constitutive FGFR1 signalling in LMP1 expressing nasopharyngeal epithelial cells.

Using conventional IHC, 12 of 42 NPC samples found to express detectable levels of LMP1 also expressed high levels of phosphorylated FGFR1. However, among thirty LMP1-negative NPC tumours, twelve displayed high levels of phosphorylated FGFR1. In this regard, mechanisms, in addition to LMP1, may be responsible for FGFR1 activation. Another possibility is that the expression of LMP1 protein in the majority of NPC samples is below the level of detection using current methods. Using a sensitive tyramid-augmented IHC method, Dietz et al have found that nearly all NPC specimens with negative LMP1-staining in conventional IHC were positive for LMP1 expression [39]. Despite the extremely low levels of expression, LMP1 can have dramatic consequences on the phenotype of epithelial cells. Studies from transgenic mice have shown that LMP1 induces epithelial hyperplasia at levels that are virtually undetectable using current available detection methods [40]. Our previous study have also indicated that very small amount of LMP1, below the level of detection, are sufficient to 
activate NFKB signalling, a key pathway that contributes to LMP1-mediated cell transformation [41]. This is also reminiscent of C666-1 NPC cell line which expresses very low level of LMP1 but high level of FGF2, regulated by NFKB signalling (Supplementary Figure 3) [24]. This raises the possibility that those NPC tumours in which LMP1 protein cannot be detected may nevertheless express functionally active LMP1.

Constitutive FGFR1 activation has been shown to induce cellular transformation and promote cancer progression. Aberrant activation of FGFR1 signalling, through overexpression of FGFR1 and/or its ligands, mutations or amplification, have been documented in a variety of human malignancies [26;27;42]. In this study, we demonstrate that FGFR1 signalling contributes to LMP1-mediated cellular transformation. Inhibition of FGFR1 pathway significantly suppressed LMP1-induced cell proliferation, anchorage-independent growth, migration and invasion (Figure 5). FGFR1 inhibitors also suppressed LMP1-mediated glycolysis as they reduced both LDHA phosphorylation and activity (Figure 3). In addition, we report that FGFR1 signalling is commonly activated in NPC, and is particularly evident in cases that are LMP1 positive (Figure 4). Previous studies indicate that genetic alterations in FGFR1 and/or its ligands (FGF1 and FGF2) are rare in NPC tumours [2]. As FGFR1 activation in NPC correlates significantly with LMP1 expression (Figure 4F), LMP1-activated FGFR1 signalling may play an important role in NPC pathogenesis. The development of cancer therapeutics based on targeting FGFR signalling is an attractive approach. Currently, many kinase inhibitors with anti-FGFR activity, in addition to FGFR1 blocking antibodies are being evaluated in clinical trials. In this study, we show that FGFR1 inhibitors suppress cell proliferation and anchorage-independent growth of C666-1 NPC cells, highlighting the therapeutic potential of the FGFR1 pathway (Figure 5). Therapeutic strategies targeting FGFR1 signalling in NPC is worthy of further examination particularly in the context of metastatic disease.

\section{Acknowledgements}

This work was supported by Focus Investigation Scheme-A from the Chinese University of Hong Kong, Research Grants Council of Hong Kong - GRF (471211, 470312, 471413), CRF (CUHK8/CRF/11R) and Theme-Based Research Scheme (T12-401/13R) funding; This study was also supported by Direct Grant for Research (2014.2.003) from the Chinese University of Hong Kong anda Cancer Research UK (CRUK)-funded China Fellowship to AKF Lo, CRUK Programme Funding to AKF Lo, CW Dawson and LS Young. We thank Masaya Imoto, Keio University for providing FGFR1 promoter construct.

\section{Statement of author contributions}


AKFL, CWD and KWL designed research, AKFL, CWD, CWK, PMH, KWL performed research, AKFL, CWD LSY and KWL analysed data and drafted the manuscript. All authors discussed the findings, reviewed the data and commented on the manuscript.

\section{List of online Supporting Information}

\section{Supplementary File 1}

Additional Materials and Methods

\section{Supplementary Fig. 1}

LMP1 induces translocation of GLUT1 from intracellular vesicles to the plasma membrane. NP69-pLNSX and NP69-LMP1 cells were subject to immunofluorescence staining with GLUT1 (left panel) and counterstained with DAPI to identify the nucleus (left panel). Three pairs of representative pictures are shown. Red arrows indicate the localization of GLUT1 in intracellular membrane compartments. Yellow arrows indicate the GLUT1 staining at the plasma membrane.

\section{Supplementary Fig. 2}

\section{LMP1 alters the expression and phosphorylation of glycolytic protein in SCC12F cells}

SCC12F-pLNSX and SCC12F-LMP1 cells were harvested and subjected to immunoblotting analysis for the indicated proteins.

\section{Supplementary Fig. 3}

\section{LMP1 increases the expression of FGFR1 and FGF2 mRNA}

NP69-pLNSX, NP69-LMP1, C666-1, SCC12F-pLNSX and SCC12F-LMP1 cells were harvested and subjected to quantitative RT-PCR analysis for FGFR1 (A) and LMP1 (B) expression. (C) The levels of FGF2 mRNA were determined in NP69-pLNSX, NP69-LMP1 and C666-1 cells. The mRNA expression of the target gene was normalized to expression of the TBP gene. Relative mRNA levels were calculated using NP69-pLNSX cells (set at 1).

\section{Supplementary Fig. 4}

\section{FGFR1 inhibitors attenuate LMP1-mediated proliferation}

SCC12F-pLNSX and SCC12F-LMP1 cells were treated with increasing doses of PD161570 (A) or SU5402 (B), as indicated, for 3 days prior to cell growth analysis.

\section{Supplementary Fig. 5}

FGFR1 inhibitors attenuate LMP1-mediated invasion 
For Boyden Chamber invasion assays, 5x10 NP69-pLNSX or NP69-LMP1 cells were plated into the chambers coated with basement membrane matrix solution. After $24 \mathrm{~h}$ incubation with indicated concentrations of FGFR1 inhibitors, invasive cells, which localise to the bottom of the membrane were stained and photographed.

\section{Supplementary Fig.6}

Schematic diagram shows the effects of LMP1 in promoting aerobic glycolysis. (1) LMP1 increases the uptake of glucose and glutamine, the concentration of intracellular glutamate and lactate, as well as the enzymatic activity of LDHA. (2) LMP1 reduces the intracellular concentration of pyruvate and the enzymatic activity of pyruvate kinase. (3) LMP1 increases the expression of HIF-1 $\alpha$, c-Myc, FGFR1, FGF2, LDHA and PDHK1, and increases the levels of phosphorylation of LDHA (Y10), PKM2 (Y105) and FGFR1 (Y653/654) to promote aerobic glycolysis.

\section{Supplementary Fig. 7}

\section{Schematic diagram showing the mechanism of LMP1-mediated upregulation of FGFR1 \& FGF2}

LMP1 activates multiple signal pathways and downstream targets to induce transcription of FGFR1 and FGF2.

\section{References}

1. Dawson CW, Port RJ, Young LS. The role of the EBV-encoded latent membrane proteins LMP1 and LMP2 in the pathogenesis of nasopharyngeal carcinoma (NPC). Semin Cancer Biol 2012; 22: 144-153.

2. Lo KW, Chung GT, To KF. Deciphering the molecular genetic basis of NPC through molecular, cytogenetic, and epigenetic approaches. Semin Cancer Biol 2012; 22: 79-86.

3. Tsao SW, Tramoutanis G, Dawson CW et al. The significance of LMP1 expression in nasopharyngeal carcinoma. Semin Cancer Biol 2002; 12: 473-487.

4. Cairns RA, Harris IS, Mak TW. Regulation of cancer cell metabolism. Nat Rev Cancer 2011; 11: 85-95.

5. Yeung SJ, Pan J, Lee MH. Roles of p53, MYC and HIF-1 in regulating glycolysis - the seventh hallmark of cancer. Cell Mol Life Sci 2008; 65: 3981-3999.

6. Chen JQ, Russo J. Dysregulation of glucose transport, glycolysis, TCA cycle and glutaminolysis by oncogenes and tumor suppressors in cancer cells. Biochim Biophys Acta 2012; 1826: 370-384.

7. Hitosugi T, Kang S, Vander Heiden MG et al. Tyrosine phosphorylation inhibits PKM2 to promote the Warburg effect and tumor growth. Sci Signal 2009; 2: ra73.

8. Hitosugi T, Chen J. Post-translational modifications and the Warburg effect. Oncogene 2014; 33: 4279-4285. 
9. Knights V, Cook SJ. De-regulated FGF receptors as therapeutic targets in cancer. Pharmacol Ther 2010; 125: 105-117.

10. Fan J, Hitosugi T, Chung TW et al. Tyrosine phosphorylation of lactate dehydrogenase A is important for NADH/NAD(+) redox homeostasis in cancer cells. Mol Cell Biol 2011; 31: 49384950.

11. Hitosugi T, Fan J, Chung TW et al. Tyrosine phosphorylation of mitochondrial pyruvate dehydrogenase kinase 1 is important for cancer metabolism. Mol Cell 2011; 44: 864-877.

12. Lo AK, Lo KW, Tsao SW et al. Epstein-Barr virus infection alters cellular signal cascades in human nasopharyngeal epithelial cells. Neoplasia 2006; 8: 173-180.

13. Lo AK, Lo KW, Ko CW et al. Inhibition of the LKB1-AMPK pathway by the Epstein-Barr virusencoded LMP1 promotes proliferation and transformation of human nasopharyngeal epithelial cells. J Pathol 2013; 230: 336-346.

14. Lo AK, Huang DP, Lo KW et al. Phenotypic alterations induced by the Hong Kong-prevalent Epstein-Barr virus-encoded LMP1 variant (2117-LMP1) in nasopharyngeal epithelial cells. Int J Cancer 2004; 109: 919-925.

15. Lo AK, Liu Y, Wang $\mathrm{XH}$ et al. Alterations of biologic properties and gene expression in nasopharyngeal epithelial cells by the Epstein-Barr virus-encoded latent membrane protein 1. Lab Invest 2003; 83: 697-709.

16. Luo W, Semenza GL. Emerging roles of PKM2 in cell metabolism and cancer progression. Trends Endocrinol Metab 2012; 23: 560-566.

17. Xiao L, Hu ZY, Dong $\mathrm{X}$ et al. Targeting Epstein-Barr virus oncoprotein LMP1-mediated glycolysis sensitizes nasopharyngeal carcinoma to radiation therapy. Oncogene 2014; 33: 45684578.

18. Sommermann TG, O'Neill K, Plas DR et al. IKKbeta and NF-kappaB transcription govern lymphoma cell survival through AKT-induced plasma membrane trafficking of GLUT1. Cancer Res 2011; 71: 7291-7300.

19. Tudor CS, Dawson CW, Eckhardt J et al. c-Myc and EBV-LMP1: two opposing regulators of the HLA class I antigen presentation machinery in epithelial cells. Br J Cancer 2012; 106: 1980-1988.

20. Kondo S, Seo SY, Yoshizaki T et al. EBV latent membrane protein 1 up-regulates hypoxiainducible factor 1alpha through Siah1-mediated down-regulation of prolyl hydroxylases 1 and 3 in nasopharyngeal epithelial cells. Cancer Res 2006; 66: 9870-9877.

21. Chen H, Hutt-Fletcher L, Cao L et al. A positive autoregulatory loop of LMP1 expression and STAT activation in epithelial cells latently infected with Epstein-Barr virus. $J$ Virol 2003; 77: 4139-4148.

22. Wakisaka N, Kondo S, Yoshizaki T et al. Epstein-Barr virus latent membrane protein 1 induces synthesis of hypoxia-inducible factor 1 alpha. Mol Cell Biol 2004; 24: 5223-5234.

23. Dirmeier U, Hoffmann R, Kilger $\mathrm{E}$ et al. Latent membrane protein 1 of Epstein-Barr virus coordinately regulates proliferation with control of apoptosis. Oncogene 2005; 24: 1711-1717.

24. Wakisaka N, Murono S, Yoshizaki T et al. Epstein-barr virus latent membrane protein 1 induces and causes release of fibroblast growth factor-2. Cancer Res 2002; 62: 6337-6344. 
25. Lo AK, To KF, Lo KW et al. Modulation of LMP1 protein expression by EBV-encoded microRNAs. Proc Natl Acad Sci U S A 2007; 104: 16164-16169.

26. Kelleher FC, O'Sullivan H, Smyth E et al. Fibroblast growth factor receptors, developmental corruption and malignant disease. Carcinogenesis 2013; 34: 2198-2205.

27. Lim SM, Kim HR, Shim HS et al. Role of FGF receptors as an emerging therapeutic target in lung squamous cell carcinoma. Future Oncol 2013; 9: 377-386.

28. Darekar S, Georgiou K, Yurchenko M et al. Epstein-Barr virus immortalization of human B-cells leads to stabilization of hypoxia-induced factor 1 alpha, congruent with the Warburg effect. PLoS One 2012; 7: e42072.

29. Fritz V, Fajas L. Metabolism and proliferation share common regulatory pathways in cancer cells. Oncogene 2010; 29: 4369-4377.

30. Wise DR, DeBerardinis RJ, Mancuso A et al. Myc regulates a transcriptional program that stimulates mitochondrial glutaminolysis and leads to glutamine addiction. Proc Natl Acad Sci U S A 2008; 105: 18782-18787.

31. Seyed M, Dimario JX. Sp1 is required for transcriptional activation of the fibroblast growth factor receptor 1 gene in neonatal cardiomyocytes. Gene 2007; 400: 150-157.

32. Kanai M, Tashiro E, Maruki $\mathrm{H}$ et al. Transcriptional regulation of human fibroblast growth factor receptor 1 by E2F-1. Gene 2009; 438: 49-56.

33. Tan NY, Khachigian LM. Sp1 phosphorylation and its regulation of gene transcription. Mol Cell Biol 2009; 29: 2483-2488.

34. Everly DN, Jr., Mainou BA, Raab-Traub N. Induction of Id1 and Id3 by latent membrane protein 1 of Epstein-Barr virus and regulation of p27/Kip and cyclin-dependent kinase 2 in rodent fibroblast transformation. J Virol 2004; 78: 13470-13478.

35. Mainou BA, Everly DN, Jr., Raab-Traub N. Unique signaling properties of CTAR1 in LMP1mediated transformation. J Virol 2007; 81: 9680-9692.

36. Mainou BA, Raab-Traub N. LMP1 strain variants: biological and molecular properties. $J$ Virol 2006; 80: 6458-6468.

37. Shi Y, Tao Y, Jiang Y et al. Nuclear epidermal growth factor receptor interacts with transcriptional intermediary factor 2 to activate cyclin D1 gene expression triggered by the oncoprotein latent membrane protein 1. Carcinogenesis 2012; 33: 1468-1478.

38. Xu Y, Shi Y, Yuan Q et al. Epstein-Barr Virus encoded LMP1 regulates cyclin D1 promoter activity by nuclear EGFR and STAT3 in CNE1 cells. J Exp Clin Cancer Res 2013; 32: 90.

39. Dietz A, Logothetis CA, Helbig M et al. Prognostic impact of EBV-related LMP-1, histologic type, and environmental factors in nasopharyngeal carcinoma in a German population. Onkologie 2004; 27: 345-350.

40. Stevenson D, Charalambous C, Wilson JB. Epstein-Barr virus latent membrane protein 1 (CAO) up-regulates VEGF and TGF alpha concomitant with hyperlasia, with subsequent up-regulation of p16 and MMP9. Cancer Res 2005; 65: 8826-8835. 
41. Lo AK, Dawson CW, Jin DY et al. The pathological roles of BART miRNAs in nasopharyngeal carcinoma. J Pathol 2012; 227: 392-403.

42. Fearon AE, Gould CR, Grose RP. FGFR signalling in women's cancers. Int J Biochem Cell Biol 2013; 45: 2832-2842.

\section{Figure Legends}

Figure 1. LMP1 promotes aerobic glycolysis. NP69-pLNSX and NP69-LMP1 cells were incubated in Keratinocyte-SFM medium supplemented with $0.2 \mathrm{ng} / \mathrm{ml}$ EGF and $30 \mu \mathrm{g} / \mathrm{ml}$ bovine pituitary extract. (A) Culture supernatants were collected at the indicated time for the determination of lactate production. (BD, H) Cell lysates were extracted for measurement of intracellular LDH activity (B), pyruvate kinase activity (C), pyruvate concentration (D) and glutamate concentration (H). (F-G) Culture supernatants were collected for measurement of glucose uptake $(F)$, and glutamine consumption $(G)$. The results are expressed by defining the control (NP69-pLNSX) as 1. Bars represent the mean \pm SEM of samples from three independent experiments. The asterisks indicate a significant difference $(* \mathrm{P}<0.05$ and $* * \mathrm{P}$ $<0.001$ ). (E) Immunofluorescence staining of GLUT1 in NP69-pLNSX and NP69-LMP1 cells. Yellow arrows indicate the localization of GLUT1 in the plasma membrane. Red arrows indicate the GLUT1 staining in intracellular membrane compartments.

\section{Figure 2. LMP1 alters the expression and activity of glycolytic proteins and increases the} expression of FGFR1. (A) The nasopharyngeal epithelial cell line NP69 was transfected with increasing amounts of LMP1 expression vector as indicated. Forty-eight hours post-transfection, cells were harvested and subjected to immunoblotting analysis for the indicated proteins. (B) NP69-pLNSX (P) and NP69-LMP1 (L) cells were incubated under either normoxic $\left(21 \% \mathrm{O}_{2}\right)$ or hypoxic $\left(3 \% \mathrm{O}_{2}\right)$ conditions for 24 hours prior to western blot analysis. (C-D, F) The NP69 cells were transferred with increasing amounts of LMP1 expression vector as indicated. Forty-eight hours post-transfection, cells were harvested and subjected to quantitative RT-PCR analysis for FGFR1 (C), LMP1 (D) and FGF2 (F). The mRNA expression of the target gene of interest was normalized to the expression of the TBP gene. Relative mRNA levels were calculated using the sample without transfected LMP1 vector (set at 1). (E) Hela cells were transfected with FGFR1 luciferase promoter vector together with various doses of LMP1 expression vector. Forty-eight hours post-transfection, cells were harvested for luciferase analysis. Luciferase activity was normalized to Renilla activity and was plotted relative to that of reporter alone (set at 1). (G). NP69-pLNSX (P) and NP69-LMP1 (L) cells were treated with 20 $\mu \mathrm{g} / \mathrm{ml}$ control IgG antibody or FGF2 neutralizing antibody in serum-free medium for 6 hours prior to western blot analysis. (H). C666-1 cells were transfected with scrambled siRNA or LMP1 siRNA. Protein lysates were harvested 48 hours after transfection for western blot analysis. Relative protein levels were calculated using control NP69-pLNSX or parental C666-1 as a reference (set at 1). 
Figure 3. Activation of FGFR1 by LMP1 facilitates aerobic glycolysis. (A) NP69-pLNSX and NP69LMP1 cells were treated with FGFR1 inhibitors, PD161570 (PD) or SU5402 (SU), for $7 \mathrm{~h}$ prior to harvesting for immunoblotting. Relative protein levels were calculated using inhibitor-free NP69pLNSX as a reference (set at 1). (B\&C) Cells were treated with FGFR1 inhibitors for $16 \mathrm{~h}$ prior to analysis of LDH activity (B) and pyruvate kinase activity (C). Relative LDH activity was calculated using inhibitor-free NP69-pLNSX as a reference (set at 1). The asterisks indicate a significant difference $(* \mathrm{P}<0.02$ and $* * \mathrm{P}<0.05)$.

Figure 4. NPC tumours display increased levels of FGFR1 phosphorylation. Immunohistochemical staining of NPC and normal nasopharyngeal epithelium specimens for Tyr 654 phosphorylated-FGFR1 (phospho-FGFR1). (A) Negative phospho-FGFR1 staining was observed in normal EBV-negative nasopharyngeal epithelia. (B) Low (score: 2) levels of FGFR1 phosphorylation in NPC tumour cells. (C) Moderate (immunoactivity score: 5) levels of phospho-FGFR1 expression in NPC tumour cells. (D \& E) Strong (immunoactivity score: 8 and 12) levels of phospho-FGFR1 staining of NPC tumour cells. (F) Graph of a dot plot showing the immunoactivity score of phospho-FGFR1 staining within the group of tumours with and without LMP1 expression $(n=42)$. The majority of the LMP1-positive NPC tumours $(11 / 12,92 \%)$ exhibited strong FGFR1 phosphorylation (score: $\geq 3$ ). The median values of each group are shown in the horizontal line. The $\mathrm{p}$ value between the two groups is shown. Black arrow: normal nasopharyngeal epithelium; Red arrow: NPC tumour cells.

Figure 5. FGFR1 inhibitors attenuate the proliferation and anchorage-independent growth of NP69-LMP1 and C666-1 cells. (A \& B) NP69-pLNSX and NP69-LMP1 cells were treated with increasing doses of PD161570 (PD) or SU5402 (SU), as indicated, for 3 days prior to cell growth analysis. For NP69-pLNSX cells, the $\mathrm{IC}_{50}$ of PD and SU are $32.8 \pm 6.0 \mu \mathrm{m}$ and $15.4 \pm 4.2 \mu \mathrm{m}$ respectively. For NP69-LMP1 cells, the $\mathrm{IC}_{50}$ of PD and SU are $25.5 \pm 6.5 \mu \mathrm{m}$ and $11.9 \pm 0.75 \mu \mathrm{m}$ respectively. (C \& D) The NPC cell line, C666-1, was treated with various doses of PD and SU for 2 to 5 days prior to cell growth analysis. (E) Soft agar growth assays were performed in duplicate for each sample and the experiment was repeated twice. The total number of colonies formed after 3 weeks from each sample was quantitated. The asterisks indicate a significant difference $(* \mathrm{P}<0.001$ and $* * \mathrm{P}<0.05)$.

Figure 6. FGFR1 inhibitors attenuate LMP1-mediated migration and invasion. (A) In wound healing assays, $0.5 \times 10^{6}$ cells were plated into inserts on a well of 24 -well plate. One day after seeding, the insert was removed to generate a defined wound field. 12 hours after incubation with the indicated concentration of FGFR1 inhibitors, cell migration was examined by phase microscopy. The wound closures were photographed and a single representative area is shown (left panel). Three individual experiments were performed and the average $\%$ of distance migration (gap difference before and after 
treatment) is shown (right panel). (B). Three-dimensional collagen gel invasion assay. Cells in the collagen gel matrix were maintained in Keratinocyte-SFM medium with indicated concentration of FGFR1 inhibitors for 5 days. The growth pattern was examined and imaged. (C). The Boyden Chamber invasion assay. $5 \times 10^{5}$ cells were plated on the chamber coated with basement membrane matrix solution. After $24 \mathrm{~h}$ incubation in the medium with indicated concentration of FGFR1 inhibitors, the invasive cells on the bottom of the membrane were stained and quantified after stain extraction. The asterisks indicate a significant difference $(* \mathrm{P}<0.05, * * \mathrm{P}<0.01)$.

\section{Fig. 7 Schematic diagram showing the effect of LMP1 on FGFR1 signaling}

LMP1 activation of FGF2/FGFR1 signaling increases the expression and/or activity of glycolysisassociated proteins and pathways, facilitating aerobic glycolysis and transformation of nasopharyngeal epithelial cells. 
Fig. 1
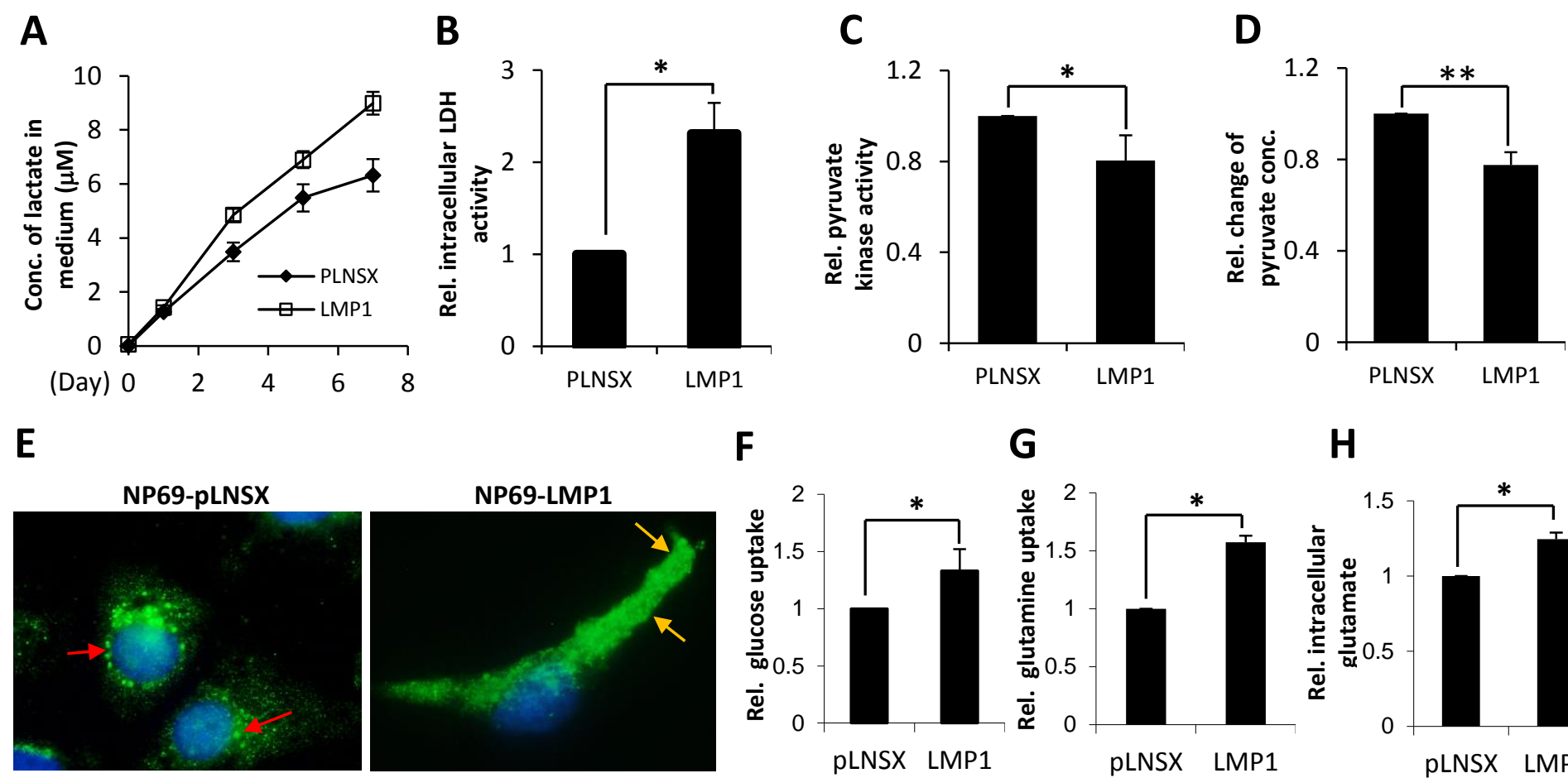

G

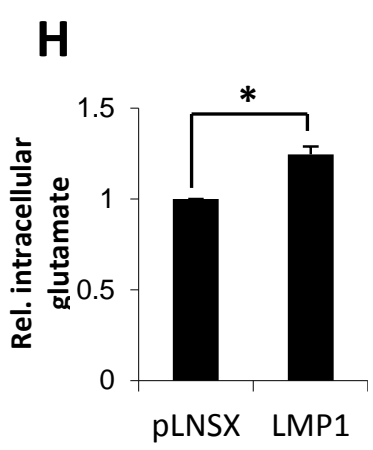




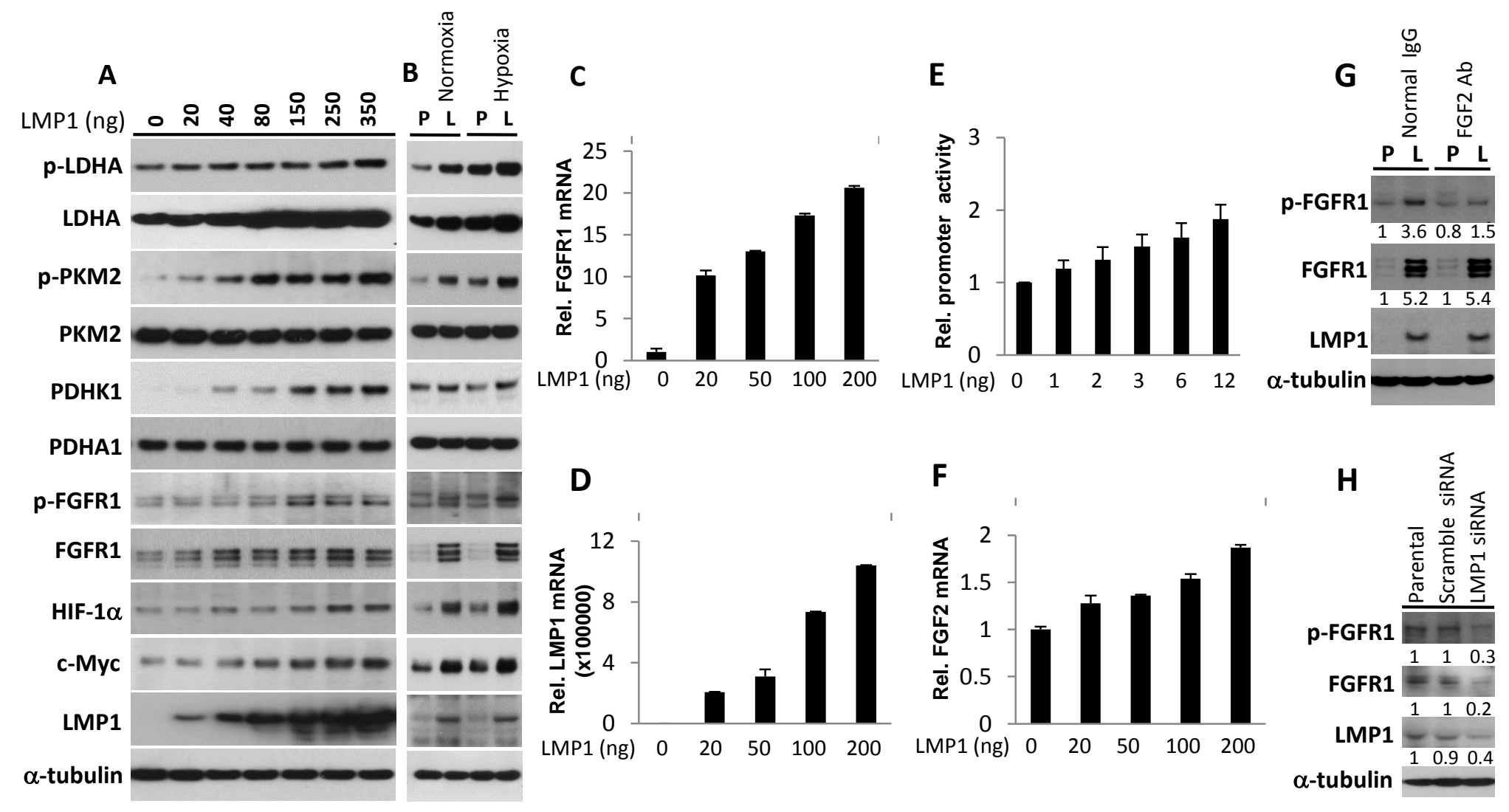


A

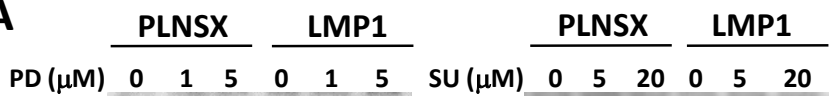
p-FGFR1

$\begin{array}{llllll}1 & 0.9 & 0.7 & 3.4 & 1.7 & 1.8\end{array}$

FGFR1

$\begin{array}{lllll}1 & 1.1 & 0.9 & 5.0 & 5.450\end{array}$

p-LDHA1 - - - - -

$\begin{array}{llllll}1 & 1 & 0.6 & 3.0 & 1.5 & 1.3\end{array}$

LDHA1

$\begin{array}{llllll}1 & 0.9 & 0.9 & 1.4 & 1.2 & 1.3\end{array}$

PDHK1 - - - - -

$$
\begin{array}{llllll}
1 & 1 & 0.7 & 2 & 1.2 & 0.7
\end{array}
$$

pPKM2 - - - -

$\begin{array}{lllllll}1 & 1 & 1 & 1.8 & 1.8 & 1.8\end{array}$

PKM2

HIF-1 $1 \alpha \begin{array}{rrrrrr}1 & 1 & 0.9 & 0.9 & 0.9 & 1\end{array}$

$\begin{array}{llllll}1 & 0.8 & 0.2 & 2.8 & 0.4 & 0.2\end{array}$

p-AKT - - - -

$\begin{array}{llllll}1 & 0.9 & 0.7 & 2.9 & 2.5 & 1.4\end{array}$

p-MAPK - - - -

$\begin{array}{llllll}1 & 0.3 & 0.2 & 2.4 & 2.3 & 0.9\end{array}$ LMP1

$\alpha$-tubulin $---\infty=$

$\begin{array}{llllll}1 & 1 & 1 & 1.6 & 1.6 & 1.4\end{array}$ $\begin{array}{llllll}1 & 1 & 1 & 1.1 & 1 & 1 \\ -\infty & & & \end{array}$

$\begin{array}{llllll}1 & 0.6 & 0.2 & 3 & 0.4 & 0.1\end{array}$

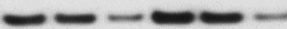

$\begin{array}{llllll}1 & 0.9 & 0.5 & 1.5 & 1.2 & 0.4\end{array}$

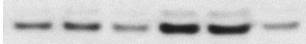

$\begin{array}{llllll}1 & 0.9 & 0.4 & 2 & 1.8 & 0.5\end{array}$

$-1-$
B

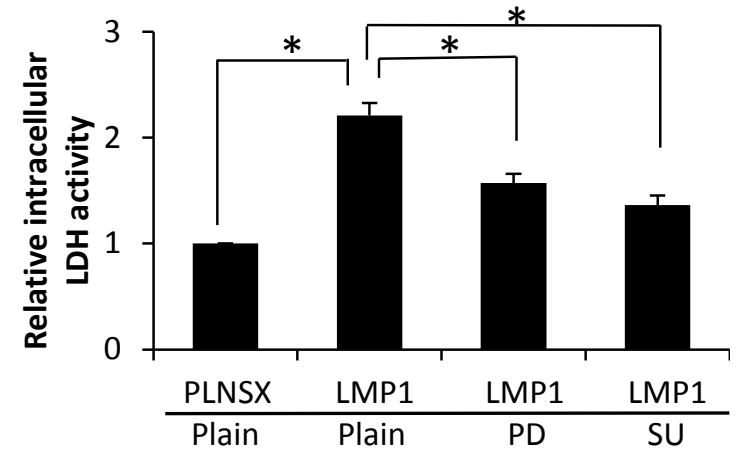

C

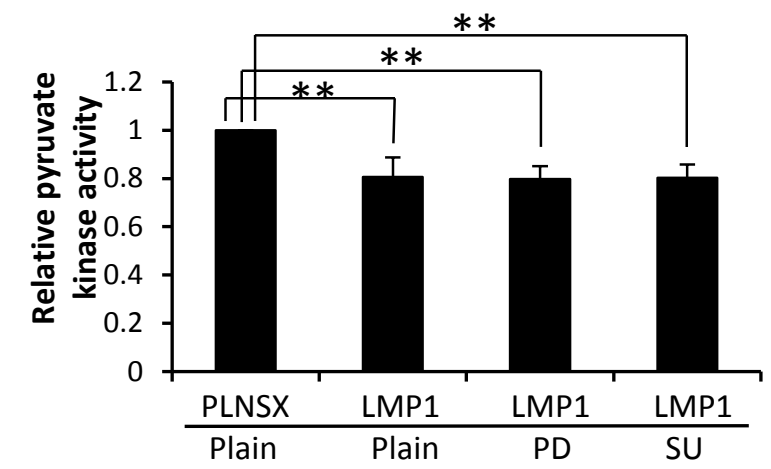




\section{Tyr 654 phosphorylated-FGFR1}

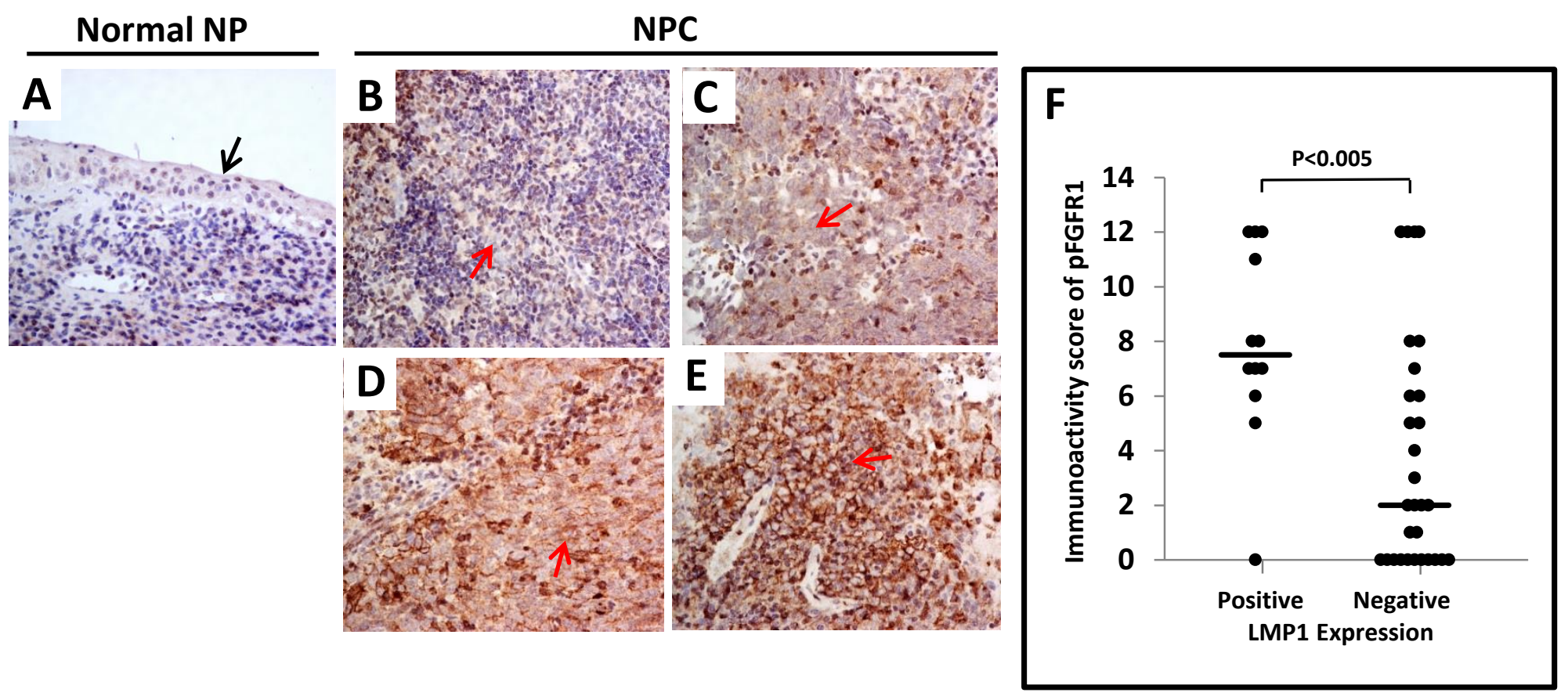



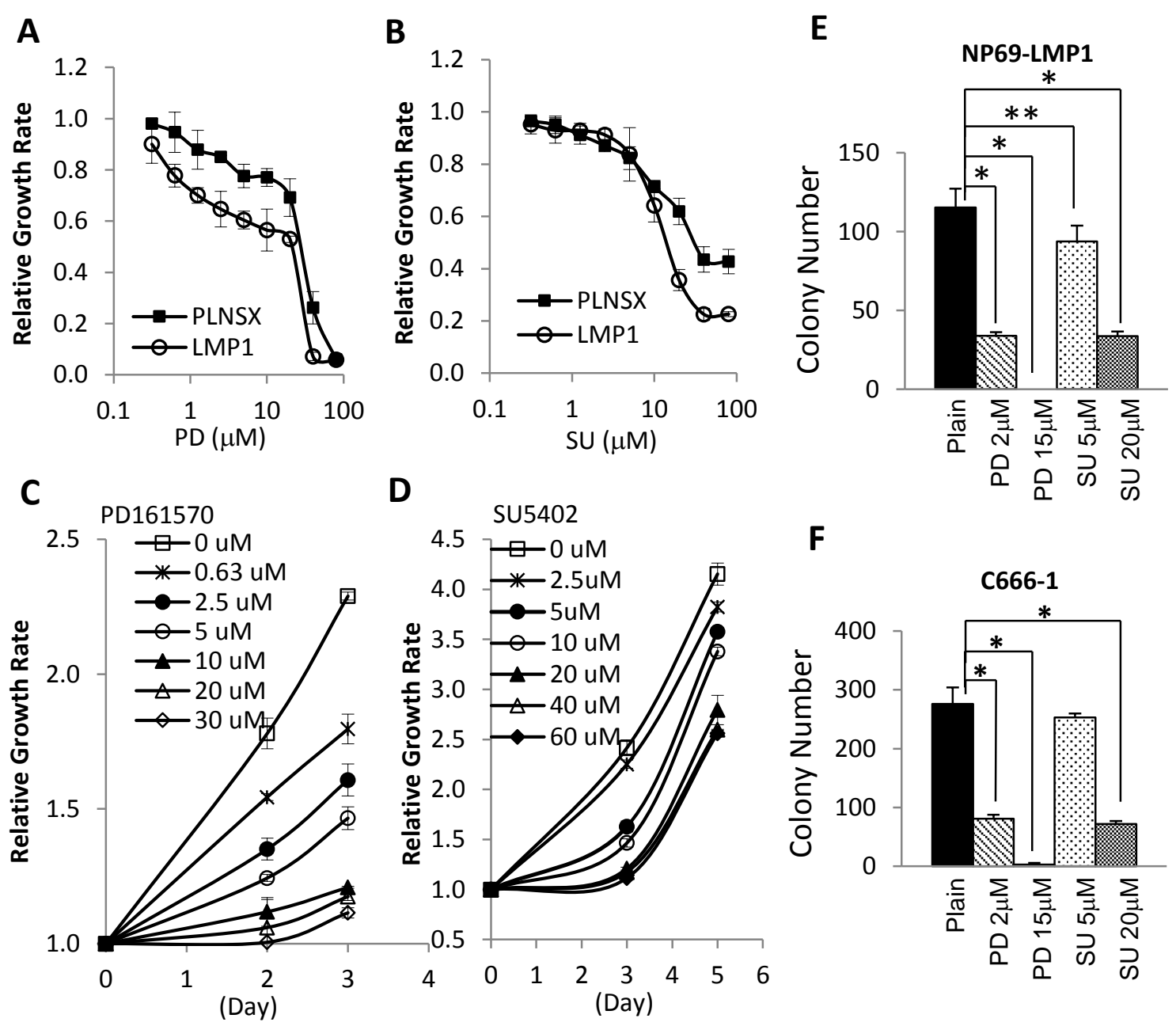

F

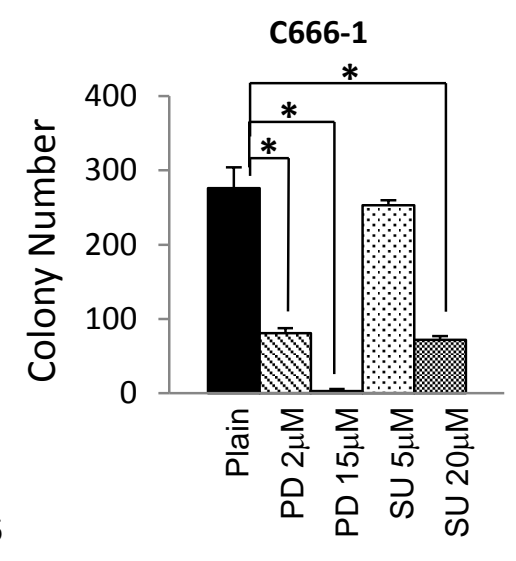


Fig. 6
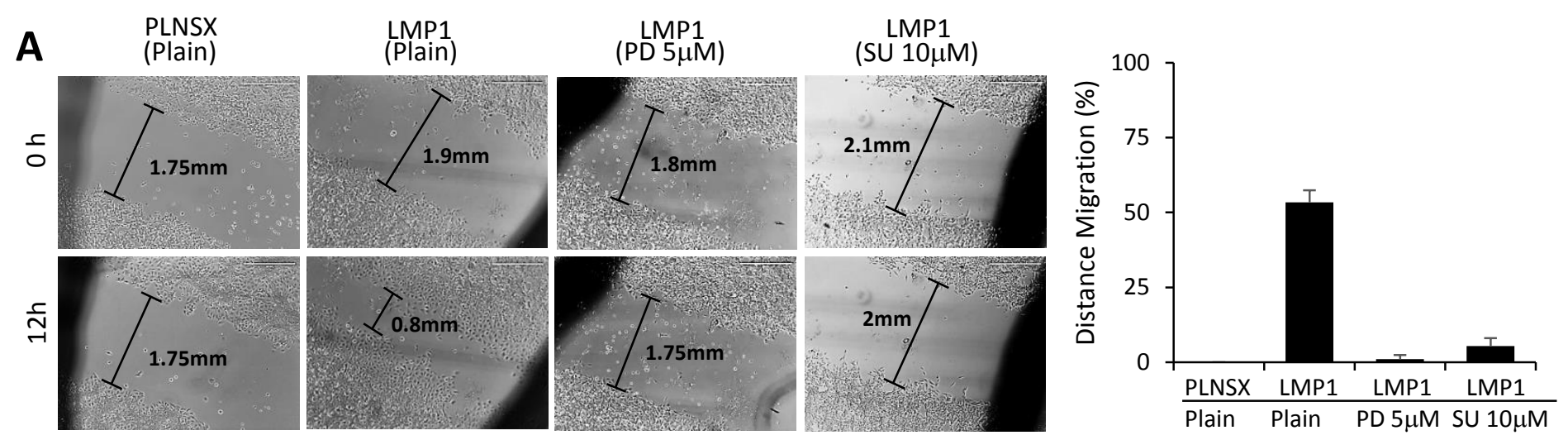

B

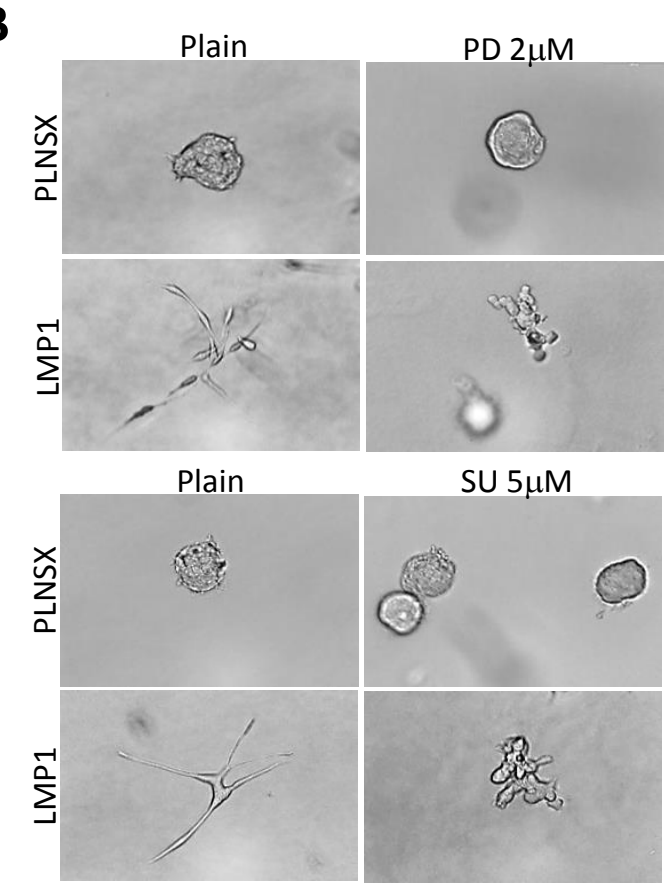

C

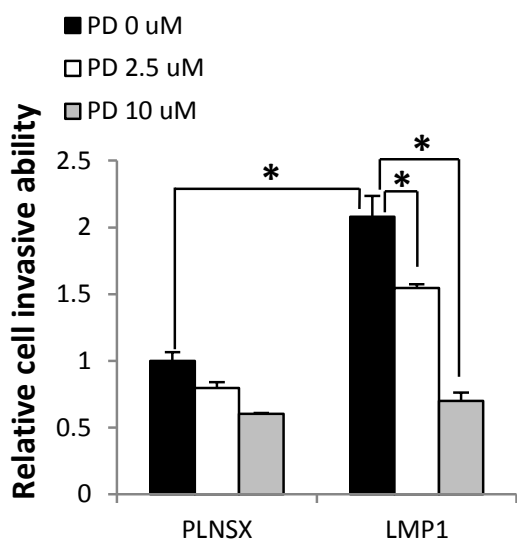

\section{- SU 0 UM}

口SU 5 UM

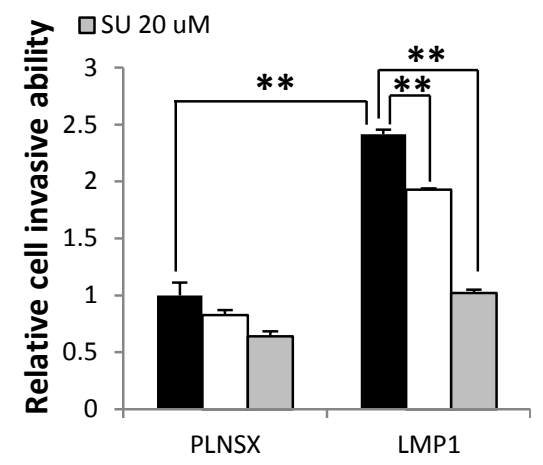




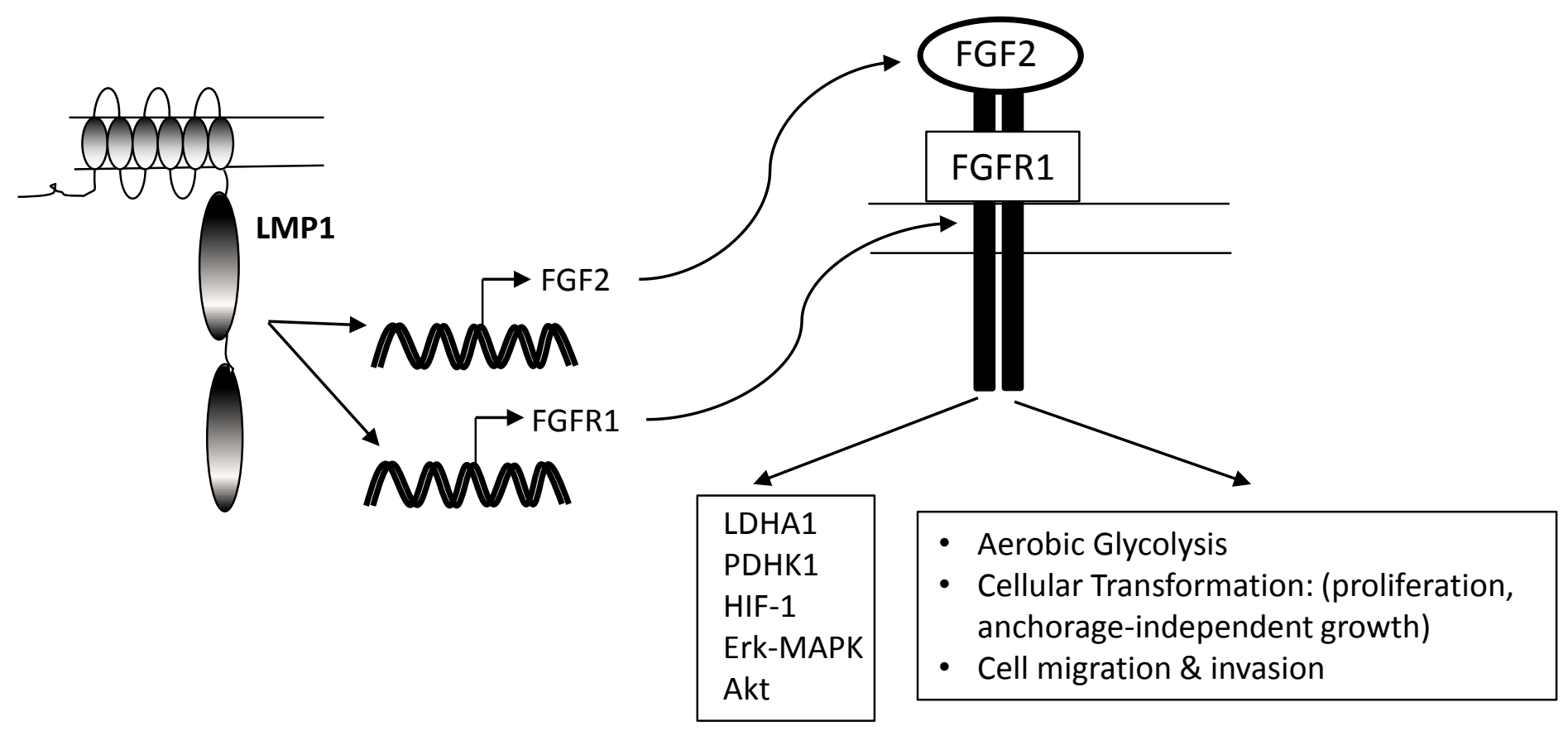


NP69-pLNSX

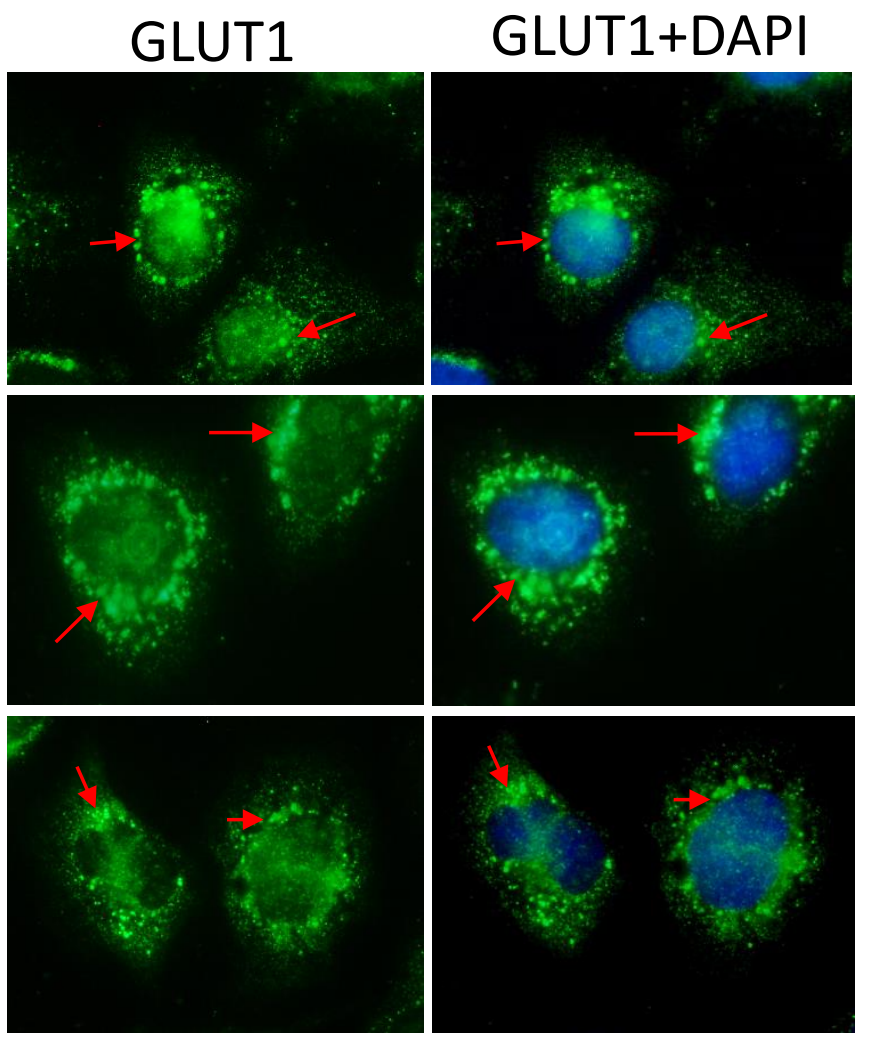

NP69-LMP1

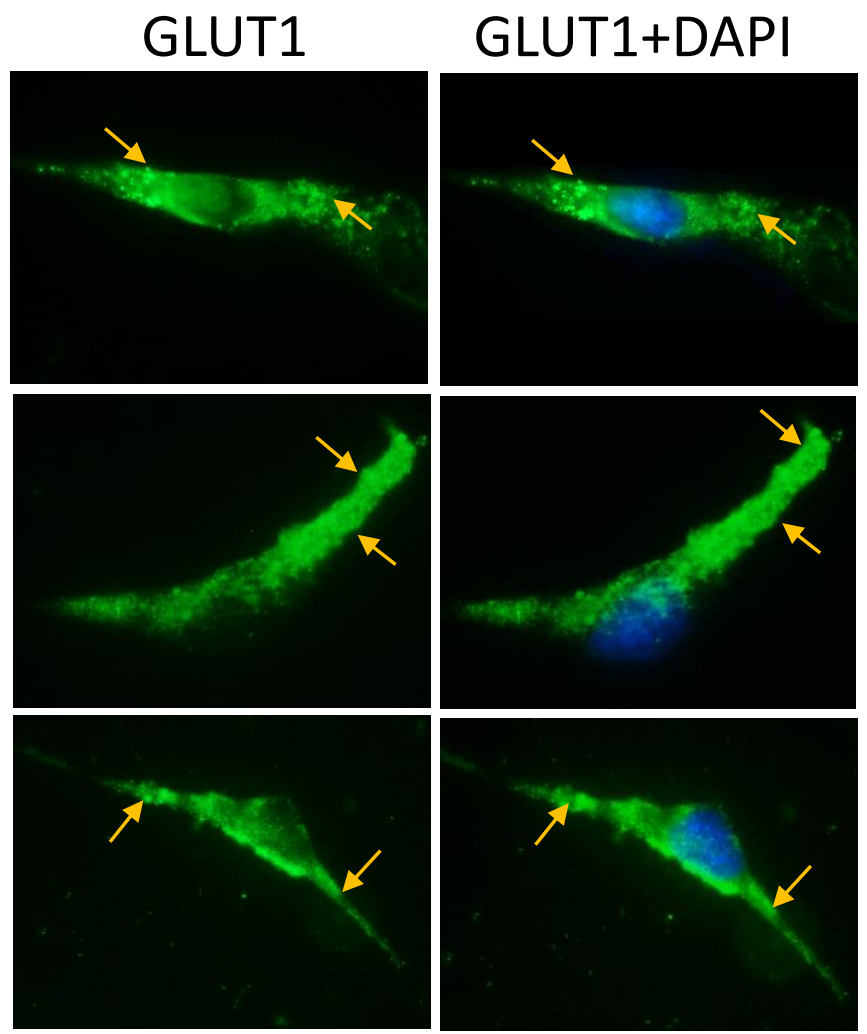

Supplementary Fig. 1

LMP1 induces translocation of GLUT1 from intracellular vesicles to the plasma membrane. NP69-pLNSX and NP69-LMP1 cells were subject to immunofluorescence staining with GLUT1 (left panel) and counterstained with DAPI to identify the nucleus (left panel). Three pairs of representative pictures are shown. Red arrows indicate the localization of GLUT1 in intracellular membrane compartments. Yellow arrows indicate the GLUT1 staining at the plasma membrane. 


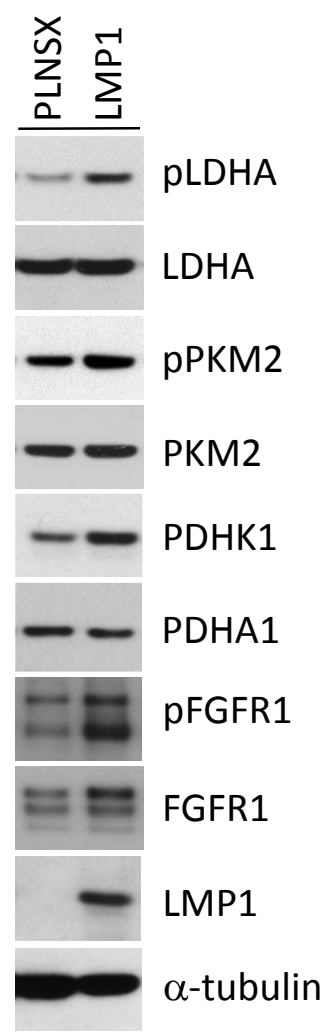

\section{Supplementary Fig. 2}

LMP1 alters the expression and phosphorylation of glycolytic protein in SCC12F cells. SCC12FpLNSX and SCC12F-LMP1 cells were harvested and subjected to immunoblotting analysis for the indicated proteins. 
A

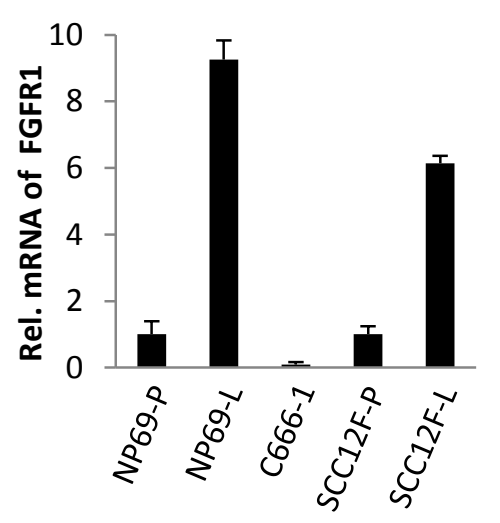

B

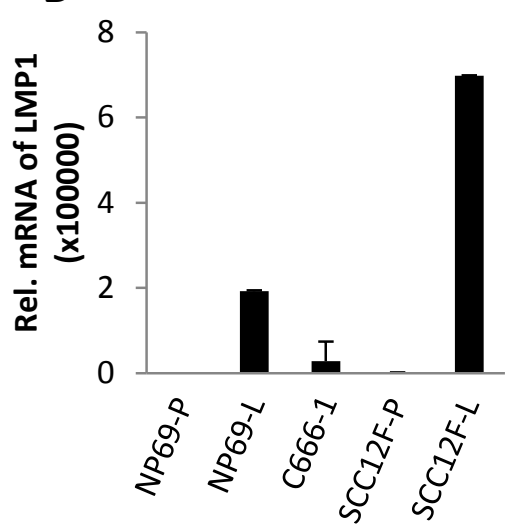

C

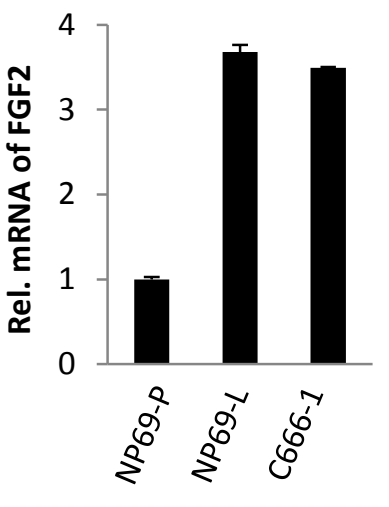

\section{Supplementary Fig. 3}

LMP1 increases the expression of FGFR1 and FGF2 mRNA. NP69-pLNSX, NP69-LMP1, C666-1, SCC12F-pLNSX and SCC12F-LMP1 cells were harvested and subjected to quantitative RT-PCR analysis for FGFR1 (A) and LMP1 (B) expression. (C) The levels of FGF2 mRNA were determined in NP69-pLNSX, NP69-LMP1 and C666-1 cells. The mRNA expression of the target gene was normalized to expression of the TBP gene. Relative mRNA levels were calculated using NP69-pLNSX cells (set at 1). 
A

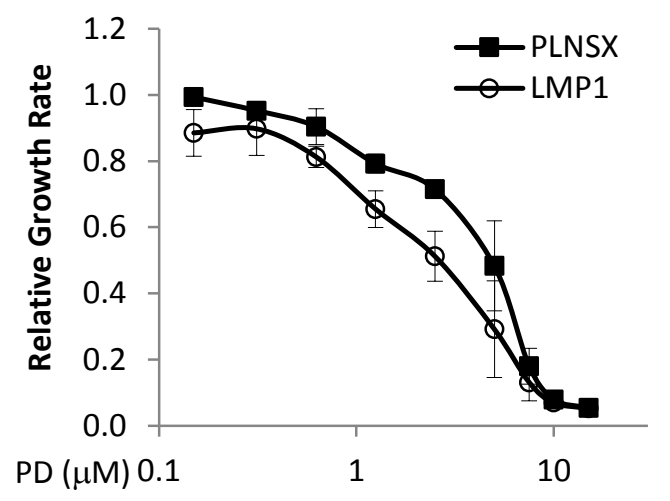

B

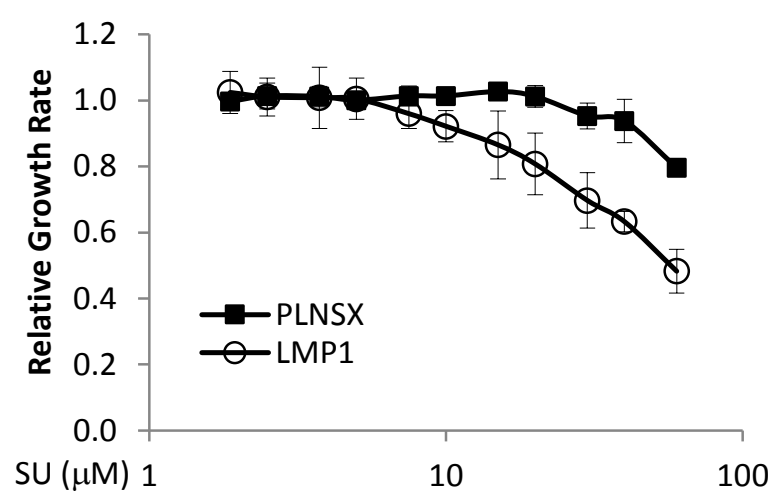

Supplementary Fig. 4

FGFR1 inhibitors attenuate LMP1-mediated proliferation. SCC12F-pLNSX and SCC12FLMP1 cells were treated with increasing doses of PD161570 (A) or SU5402 (B), as indicated, for 3 days prior to cell growth analysis. 

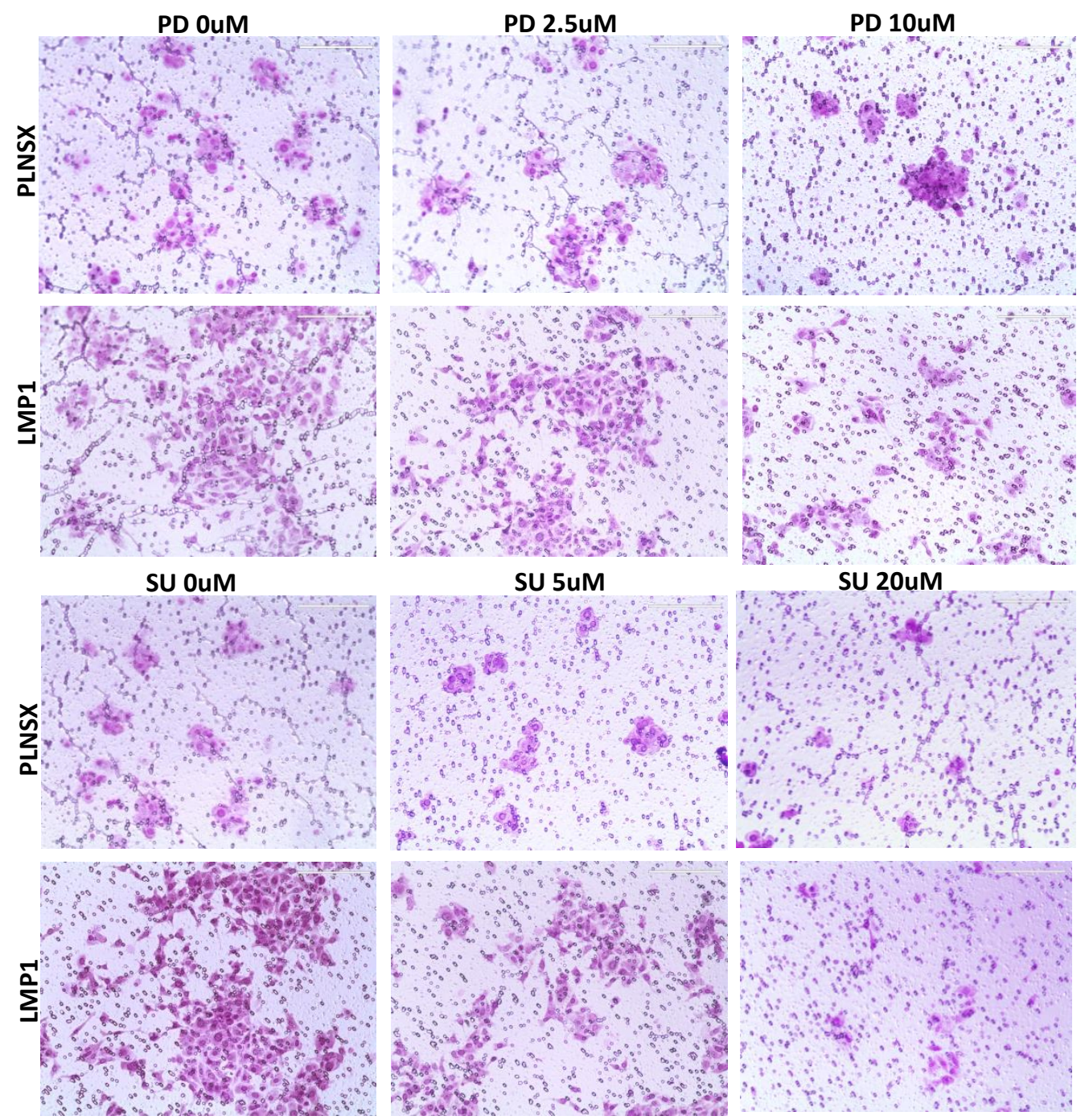

\section{Supplementary Fig. 5}

FGFR1 inhibitors attenuate LMP1-mediated invasion. For Boyden Chamber invasion assays, $5 \times 10^{5}$ NP69-pLNSX or NP69-LMP1 cells were plated into the chambers coated with basement membrane matrix solution. After $24 \mathrm{~h}$ incubation with indicated concentrations of FGFR1 inhibitors, invasive cells, which localise to the bottom of the membrane were stained and photographed. 


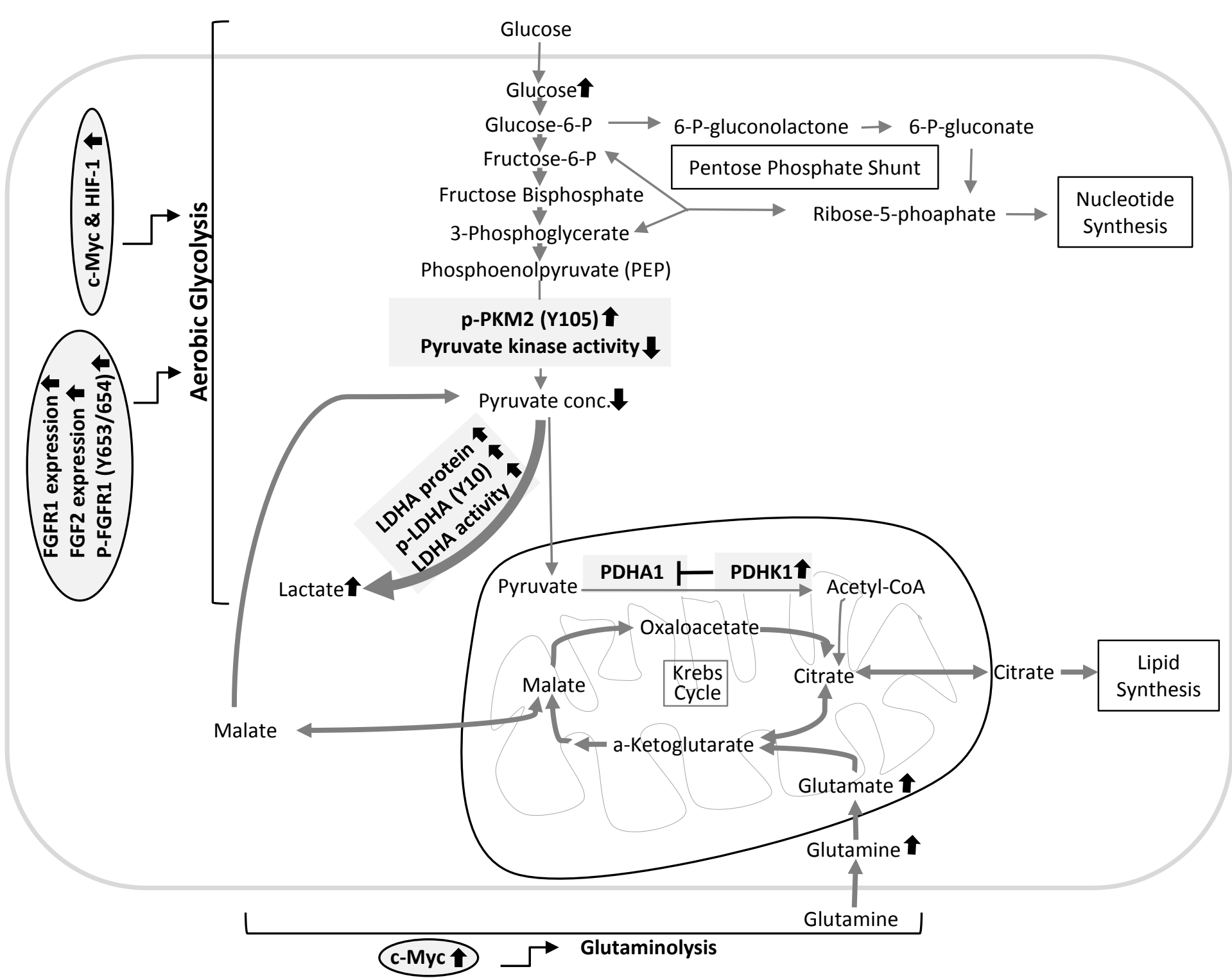

\section{Supplementary Fig.6}

Schematic diagram shows the effects of LMP1 in promoting aerobic glycolysis. (1) LMP1 increases the uptake of glucose and glutamine, the concentration of intracellular glutamate and lactate, as well as the enzymatic activity of LDHA. (2) LMP1 reduces the intracellular concentration of pyruvate and the enzymatic activity of pyruvate kinase. (3) LMP1 increases the expression of HIF-1a, c-Myc, FGFR1, FGF2, LDHA and PDHK1, and increases the levels of phosphorylation of LDHA1 (Y10), PKM2 (Y105) and FGFR1 (Y653/654) to promote aerobic glycolysis. 


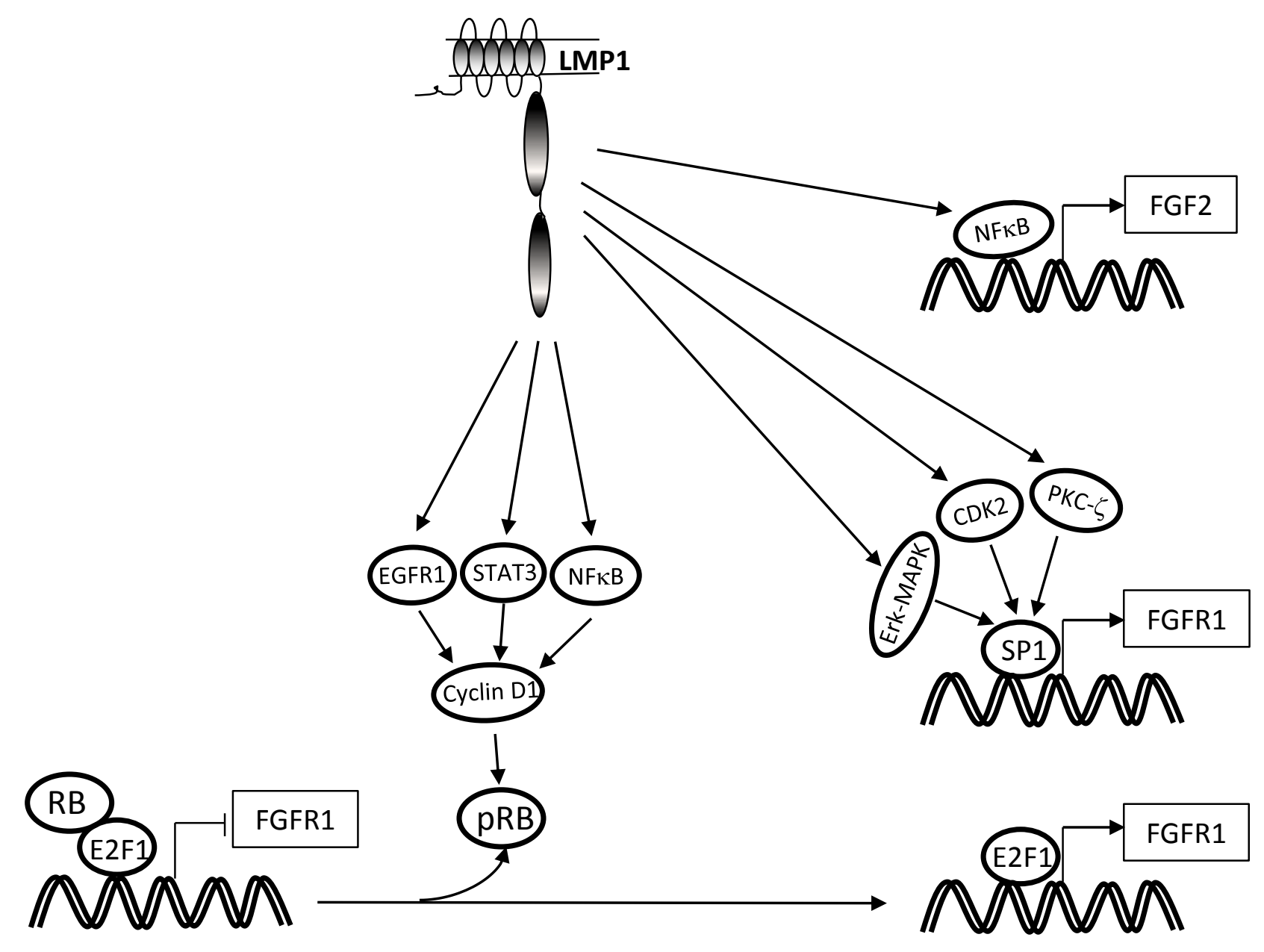

Supplementary Fig. 7

Schematic diagram showing the mechanism of LMP1-mediated upregulation of FGFR1 \& FGF2. LMP1 activates multiple signal pathways and downstream targets to induce transcription of FGFR1 and FGF2[1, 24, 31-38]. 